\title{
Interpreting the Atmospheric Circulation Trend during the Last Half of the Twentieth Century: Application of an Adjoint Model
}

\author{
SIMON BLESSING \\ Meteorologisches Institut, Universität Hamburg, Hamburg, Germany
}

RichARD J. GREATBATCH

Department of Oceanography, Dalhousie University, Halifax, Nova Scotia, Canada, and IFM-GEOMAR, Kiel, Germany

Klaus Fraedrich AND Frank LunKeit

Meteorologisches Institut, Universität Hamburg, Hamburg, Germany

(Manuscript received 12 April 2007, in final form 20 December 2007)

\begin{abstract}
A tangent linear adjoint for a low-resolution dynamical model of the atmosphere is used to derive the optimal forcing perturbations for all state variables such that after a specified lead time the model response has a given projection, in terms of an energy norm, on the pattern associated with the 51-yr trend in the Northern Hemisphere winter tropospheric circulation, 1948/49-1998/99. A feature of the derived forcing sensitivity is a Rossby wave-like feature that emanates from the western tropical Pacific and is associated with the deepening of the Aleutian low, whereas an annular pattern in the forcing sensitivity in the uppermost model level is shown to be associated with the pattern of the trend over the Euro-Atlantic/Asian sectors, including the upward trend in the North Atlantic Oscillation index. The authors argue that the Rossby wave-type feature is consistent with studies that have argued a role for the upward trend in tropical sea surface temperature during the 51-yr period. On the other hand, the authors interpret the annular pattern in the forcing sensitivity as being consistent with studies that have argued that the trend over the Euro-Atlantic sector was associated with influences from the stratosphere. In particular, a nonlinear model driven by the optimal forcing perturbation applied only to the top model level is successful at reproducing the trend pattern with the correct amplitude in the Euro-Atlantic sector, but implies a trend over the North Pacific toward a weaker Aleutian low, contrary to what was observed but similar to the spatial pattern associated with the northern annular mode. These results show that the adjoint approach can shed light on previous apparently different interpretations of the trend. The study also presents a successful application of a tangent linear adjoint model to a climate problem.
\end{abstract}

\section{Introduction}

Figure 1 shows the trend in Northern Hemisphere winter [December-February (DJF)] 500-hPa geopotential height (hereafter Z500) during the last half of the twentieth century (1948/49-1998/99) taken from National Centers for Environmental Prediction (NCEP) reanalysis data. Although there are some variations in details between different reanalysis products, the basic features are robust, in particular the deepening trend

Corresponding author address: Simon Blessing, FastOpt GmbH, Schanzenstraße 36, D-20357 Hamburg, Germany.

E-mail: simon.blessing@zmaw.de for both the Aleutian and Icelandic lows. The latter was associated with an upward trend in the North Atlantic Oscillation (NAO) index (e.g., Hurrell 1996; Hurrell et al. 2003), which itself has been the subject of much speculation regarding the possible role of anthropogenic forcing (e.g., Wunsch 1999; Greatbatch 2000; Feldstein 2002; Gillett et al. 2003). The pattern of the trend projects strongly onto the so-called cold oceanwarm land (COWL) pattern (see Lu et al. 2004; Wu and Straus 2004) identified by Wallace et al. (1996), and it has been suggested that the trend corresponds to an increased occupancy of the circulation regime associated with COWL (Corti et al. 1999).

Currently, there are two competing hypotheses as to 


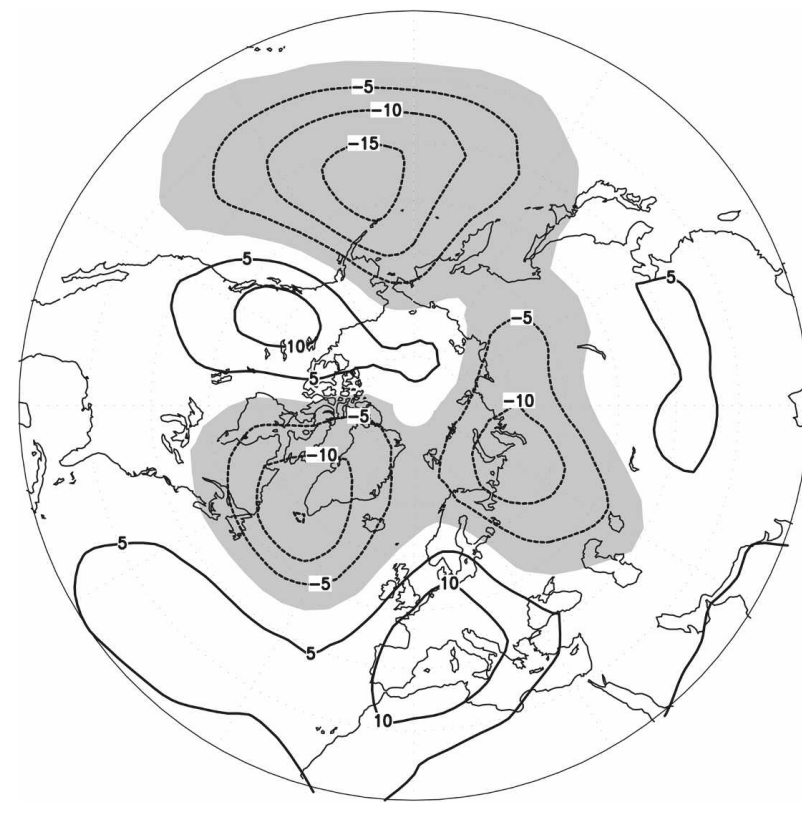

FIG. 1. Linear trend in $500-\mathrm{hPa}$ height over 51 winters of NCEP-NCAR reanalysis data. Contours are every $5 \mathrm{~m}(10 \mathrm{yr})^{-1}$ and negative regions are shaded. All centers are significant on the $5 \%$ level under the assumption that winter means are independent and normally distributed.

the origins of the circulation trend, both of which could involve some contribution from anthropogenic forcing. The first concerns the upward trend in tropical ocean sea surface temperature (SST). In particular, Hoerling et al. (2001) showed that the National Center for Atmospheric Research (NCAR) Community Climate Model, version 3 (CCM3), run with the time series of the observed SST at the lower boundary, reproduces the observed trend in Northern Hemisphere winter Z500 in the ensemble mean sense, but with the amplitude reduced by half. Their work further pointed to the importance of forcing from the tropical Pacific and Indian Oceans, and indeed there was a marked shift in tropical convection eastward in the tropical Pacific associated with the climate shift that took place around 1976 (e.g., Trenberth et al. 2002; Deser et al. 2004). Nevertheless, Hoerling et al. (2004) and Hurrell et al. (2004) have gone on to argue an important role for the upward trend in SST in the tropical Indian Ocean (see also Bader and Latif 2003) and have noted that forcing the model with only the linear trend in SST at the lower boundary is successful at capturing the deepening trend of the Icelandic low, but that the deepening trend of the Aleutian low depends in their model on including the interannual variability of the tropical ocean SST. These authors have further argued that the discrepancy in amplitude between the trend in Z500 in their model simu- lations and in reality can be accounted for by a coincidence between the forced signal (captured by the ensemble mean) and internal variability of the climate system. Work by other authors has also pointed to the importance of forcing from the tropics, notably Lu et al. (2004) and Kucharski et al. (2006). The latter argue that the western tropical Pacific warm pool region played an important role in forcing the trend.

There has also been much speculation about the possible role of the stratosphere in the dynamics of the Northern Hemisphere winter circulation trend. This is because the strengthening of the circumpolar vortex was not confined to the troposphere alone, but also occurred in the stratosphere (e.g., Thompson et al. 2000). Furthermore, there is mounting evidence that circulation changes in the winter stratosphere can indeed affect the troposphere, with the changes in the stratosphere leading those in the troposphere on a time scale of days to months (e.g., Baldwin and Dunkerton 1999; Polvani and Kushner 2002; Gillett and Thompson 2003; Gillett et al. 2003; Charlton et al. 2004; Jung and Barkmeijer 2006). Indeed, Scaife et al. (2005) have pointed out that models driven by the observed time series of SST at the lower boundary consistently fail to capture the correct amplitude of the trend, some models doing much worse in this respect than Hoerling et al. (2001). Scaife et al. take a different approach and impose a trend on the stratospheric circulation in the Hadley Centre Atmospheric Model, version 3 (AM3), comparable to that observed in the stratospheric circulation, and find that the model successfully reproduces the trend in the tropospheric circulation over the EuroAtlantic sector with the correct amplitude, even though there is no anomalous SST forcing. These results suggest an important role for the stratosphere in the dynamics of the trend, at least over the North Atlantic sector. Nevertheless, there is still the question of the origin of the changes in the stratospheric circulation. In the case of the Northern Hemisphere, there is no really conclusive evidence that this is related to changes in the chemical composition of the stratosphere (e.g., ozone; Gillett et al. 2003), in which case the trend in the stratospheric circulation may well originate from the troposphere. Evidence that there is an influence from El Niño events on the stratosphere has been presented by Taguchi and Hartmann (2006), suggesting a possible link, once again, to tropical ocean SST.

To gain more clarity about the different hypotheses, a method is required that identifies forcing perturbations that can excite a given climate response (in our case the pattern associated with the atmospheric circulation trend during the last half of the twentieth century). For nonlinear systems like the atmosphere, this is 
a nontrivial task and a variety of methods exist, all of which have in common that they attempt to estimate a linear operator that links changes in climate to changes in forcing. The simplest and computationally most expensive approach is the execution of many forward runs using different small forcing perturbations, for example, at each grid point, in order to estimate a complete set of Greens functions (an example is given by Branstator 1985). Another approach is based on a modified fluctuation-dissipation theorem (Gritsun and Branstator 2007 and references therein) and estimates the climate response to a (weak) forcing perturbation by using the covariances and lag covariances of fluctuations of the undisturbed system. This approach relies on historical or synthetic data and involves the computation and handling of correlation matrices of what is usually a very high-dimensional state space. A related study is that of Penland (1989) based on the assumption that the atmosphere can be optimally modeled by a linear Markov process. Penland's approach assumes that the underlying system dynamics is linear and noise driven, whereas the fluctuation-dissipation approach of Gritsun and Branstator has the advantage that it does not require this assumption. A similar approach is that of Branstator and Haupt (1998), who construct a linear empirical model by seeking a best fit between the state vector of a fully nonlinear dynamical model and its time tendency.

Our approach is different again and uses a tangent linear adjoint for a nonlinear dynamical model applied to both model-generated (state space) trajectories and trajectories taken from observations. The computations directly yield an estimate of the forcing perturbation that optimally excites the given climate response with a given lead time. Since the forcing perturbation is optimal (in the sense to be defined in section 2) it is not the only forcing perturbation that can excite the climate response and, in the case of the trend, it is not necessarily the forcing perturbation that actually led to the trend during the last half of the twentieth century. Rather, our approach indicates the most effective way of exciting a climate response that is similar to the trend pattern and, hopefully, throws light on the different interpretations of the trend noted earlier. A general discussion of the approach can be found in Eyink et al. (2004). A related issue is the question of the relevance to the climate problem of optimal forcing perturbations derived using a linear adjoint model with a lead time of days. Previous works - for example, Corti and Palmer (1997) and, more recently, Jung and Barkmeijer (2006) - suggest that forcing derived using a tangent linear adjoint, and applied to a fully nonlinear dynamical model, does indeed lead to a systematic forcing of the target pattern, an issue discussed further in this paper and also by Iversen et al. (2008). Iversen et al., like us, use a tangent linear adjoint to determine optimal forcing perturbations for the COWL pattern (to which the pattern of the trend is closely related). Their basic model is the European Centre for Medium-Range Weather Forecasts (ECMWF) operational model, and they are only able to determine the optimal forcing with a lead times of 4 days. In our study, because of the much coarser resolution of our model, we are able to extend the lead out as far as 14 days (the results we show use 12 days lead time).

The dynamical model used to derive the tangent linear adjoint is essentially the same as that used by Lu et al. (2004). Lu et al. had earlier argued that the trend can be captured by a model linearized about the climatological winter mean state and that the important forcing originates in the tropical Indo-Pacific region. We therefore begin by using the adjoint for a model linearized about the winter climatological mean state, and then go on to use the adjoint tangent linear version of the model linearized about both observed and modelgenerated trajectories.

We begin in section 2 by describing the model setup and the adjoint technique. Section 3 presents the results, and section 4 provides a summary and discussion.

\section{Description of the adjoint model}

We take as our starting point the model of Hall (2000), previously used by Lu et al. (2004) to study the trend. The dynamical core of the Hall model is the semispectral model of Hoskins and Simmons (1975) and is essentially the same as employed in the Portable University Model of the Atmosphere (PUMA; Fraedrich et al. 2005) developed at the University of Hamburg. PUMA is used for all the model runs presented here (the dynamical cores of the two models differ only in the spectral truncation: while PUMA uses triangular, the Hall model uses a "jagged" truncation). The Hall model has a flat bottom, a horizontal resolution of T21, and a vertical resolution of five $\sigma$ levels. The model solves the dry primitive equations on a sphere and employs empirical forcing for all state variables derived from daily mean data for the Northern Hemisphere winter season taken from the NCEPNCAR reanalysis (the technique is an extension to the primitive equations of that applied by Marshall and Molteni 1993 to a quasigeostrophic model). The model exhibits a realistic climate (Hall 2000) and also reproduces the principal modes of variability exhibited by the Northern Hemisphere winter troposphere (e.g., the North Atlantic Oscillation and the Pacific-North 
America pattern; Wallace and Gutzler 1981). To study the trend, Lu et al. (2004) used forcings derived independently for each winter from 1948/49 to 1998/99. These forcings consist of climatological forcing (the average over all 51 winters) plus forcing anomalies for each winter. In the present study, we take the inverse approach. In particular, we use the adjoint model to infer the optimal forcing anomaly that can excite the pattern associated with the trend with lead times up to 14 days. The model parameters and resolution are the same as used by Hall (2000) and Lu et al. (2004). Forward model runs are carried out (see below) using a constant forcing. The tangent linear and adjoint code were generated with the aid of the Tangent Linear and Adjoint Model Compiler (TAMC; Giering and Kaminski 1998), a source-to-source compiler.

To understand how the adjoint technique works, we begin by noting that the forward model can be written as the action of a nonlinear operator $N$ on the model state $\boldsymbol{\Psi}$ plus a time-independent forcing term $\mathbf{f}$ :

$$
\frac{\partial \Psi}{\partial t}=N(\boldsymbol{\Psi})+\mathbf{f}
$$

Linearizing about a given (possibly time dependent) reference state $\boldsymbol{\Psi}_{0}(t)$ with respect to model state and forcing yields the tangent linear model with forcing. The tangent linear model predicts the first-order development of perturbations of the original model:

$$
\left.\frac{\partial \delta \boldsymbol{\Psi}}{\partial t}\right|_{\boldsymbol{\Psi}_{0}(t)}=\left.\frac{\partial N}{\partial \boldsymbol{\Psi}}\right|_{\boldsymbol{\Psi}_{0}(t)} \delta \boldsymbol{\Psi}+\delta \mathbf{f} .
$$

Integrating Eq. (2) from time $t=t_{1}$ to $t_{2}$, with the forcing perturbation $\delta \mathbf{f}$ set to zero, gives an operator $\mathbf{R}_{t_{1}}^{t_{2}}$ (called the resolvent or propagator, $\delta \boldsymbol{\Psi}_{t_{2}}=\mathbf{R}_{t_{1}}^{t_{2}} \delta \boldsymbol{\Psi}_{t_{1}}$ ), which is linear with respect to the initial state perturbation $\delta \boldsymbol{\Psi}_{t_{1}}$, but still depends nonlinearly on the reference state $\boldsymbol{\Psi}_{0}(t)$. It propagates a perturbation $\delta \boldsymbol{\Psi}_{t_{1}}$ at $t=t_{1}$ to $\delta \boldsymbol{\Psi}_{t_{2}}$ at $t=t_{2}$ and can be expressed as $\mathbf{R}_{t_{1}}^{t_{2}}=\partial \boldsymbol{\Psi}_{t_{2}}{ }^{\prime}$ $\partial \boldsymbol{\Psi}_{t_{1}}$. The solution of Eq. (2) with forcing then takes the form

$$
\delta \boldsymbol{\Psi}_{t_{2}}=\mathbf{R}_{t_{1}}^{t_{2}} \delta \boldsymbol{\Psi}_{t_{1}}+\int_{t_{1}}^{t_{2}} \mathbf{R}_{t}^{t_{2}} \delta \mathbf{f} d t
$$

[compare, for example, with Eq. (6) in Barkmeijer et al. 2003]. Setting the initial perturbation of the model state $\delta \boldsymbol{\Psi}_{t_{1}}$ to zero and replacing the integral with a sum over the actually performed model time steps leads to

$$
\delta \boldsymbol{\Psi}_{t_{2}}=\sum_{t=t_{1}}^{t_{2}} \mathbf{R}_{t}^{t_{2}} \delta \mathbf{f} \Delta t .
$$

Thus the effect of the forcing is expressed as the accumulated action of the linear operator $\mathbf{R}_{t}^{t_{2}}$ on the small model perturbation $\delta \mathbf{f} \Delta t$ at each time step.

In the following it is the adjoint $\mathbf{R}_{t}^{t^{*}{ }^{*}}$ of $\mathbf{R}_{t}^{t_{2}}$ that is used to determine the optimal constant anomalous forcing $\delta \mathbf{f}_{\text {opt }}$ required to excite a targeted state perturbation $\delta \boldsymbol{\Psi}_{T}$ with lead time $t_{2}-t_{1}$. By definition, an adjoint operator satisfies the equation $\left\langle\mathbf{R} \boldsymbol{\Psi}_{a}, \boldsymbol{\Psi}_{b}\right\rangle=\left\langle\boldsymbol{\Psi}_{a}\right.$, $\left.\mathbf{R} * \boldsymbol{\Psi}_{b}\right\rangle$ for some scalar product. Here $\langle\cdot, \cdot\rangle$ defines the Euclidian scalar product so that, in matrix notation, we have $\left(\mathbf{R} \boldsymbol{\Psi}_{a}\right)^{\mathrm{T}} \boldsymbol{\Psi}_{b}=\boldsymbol{\Psi}_{a}^{\mathrm{T}}\left(\mathbf{R}^{\mathrm{T}} \boldsymbol{\Psi}_{b}\right)$.

We now introduce a projection index $I$ of the model state $\boldsymbol{\Psi}$ on the observed trend pattern $\delta \boldsymbol{\Psi}_{T}$. Its variation $\delta I$ with respect to an evolved model state perturbation $\delta \boldsymbol{\Psi}_{2}$ is given by

$$
\delta I=\left(\delta \boldsymbol{\Psi}_{t_{2}} ; \delta \boldsymbol{\Psi}_{T}\right) .
$$

The scalar product $(\cdot ; \cdot)$ is defined as the area- $(\mathbf{W})$ and energy-weighted (E) Euclidean scalar product $\langle\cdot$, WE $\cdot\rangle$. The energy weighting attempts to make the contributions of the different physical variables of the model state vector comparable in the contribution to the norm (see the sensitivity measure in Langland et al. 2002 and Barkmeijer et al. 2003 for further discussion of this issue). The entries of the matrix $\mathbf{E}$ are derived from the linearized total energy norm of a perturbation to an isothermal, resting atmosphere with horizontally invariant surface pressure (Talagrand 1991; Errico 2000). It is diagonal and its elements are defined in terms of the Euclidian scalar product $\langle\cdot, \cdot\rangle$ by the equation

$$
\begin{aligned}
\left\langle\delta \boldsymbol{\Psi}_{1}, \mathbf{W E} \delta \boldsymbol{\Psi}_{2}\right\rangle= & \frac{1}{2 g} \sum_{\lambda, \phi}\left[\left(\sum_{\sigma}\left\{\left[\delta u_{1}(\sigma) \delta u_{2}(\sigma)+\delta v_{1}(\sigma) \delta v_{2}(\sigma)+\frac{c_{p}}{T_{\text {ref }}} \delta T(\sigma)_{1} \delta T(\sigma)_{2}\right] \bar{p}_{s} \Delta \sigma_{h}\right\}\right.\right. \\
& \left.\left.+T_{\text {ref }} p_{\text {ref }} R \delta \ln p_{s 1} \delta \ln p_{s 2}\right) w_{\lambda, \phi}\right] .
\end{aligned}
$$

Here $\boldsymbol{\Psi}_{i}=\left(\zeta_{i}, D_{i}, T_{i}, \ln p_{s_{i}}\right)^{\mathrm{T}}$, where $\zeta$ is vorticity, $D$ is horizontal divergence, $T$ is temperature, and $p_{s}$ is surface pressure. The horizontal velocity $\left(u_{i}, v_{i}\right)$ is a func- tion of $\zeta_{i}$ and $D_{i}$ given by $\left[(\mathbf{k} \times \nabla) \Delta^{-1} \zeta+\nabla \Delta^{-1} D\right](\mathbf{k}$ is a unit vector in the upward vertical direction), $g$ is the gravitational acceleration, $\bar{p}_{s}$ the global mean surface 
pressure, $c_{p}$ is the specific heat of dry air at constant pressure, $T_{\text {ref }}=300 \mathrm{~K}$ is a reference temperature, $\Delta \sigma_{h}$ is the distance between adjacent half levels, $p_{\text {ref }}=800$ $\mathrm{hPa}$ is a reference pressure, $R$ is the gas constant for dry air, and $w_{i, j}$ are the relative area weights. Thus, using Eqs. (4) and (5), linearity, and the adjoint propagator $\mathbf{R}_{t}^{t^{*}}$, we get

$$
\begin{aligned}
\delta I & =\left\langle\sum_{t=t_{1}}^{t_{2}} \mathbf{R}_{t}^{t_{2}} \delta \mathbf{f} \Delta t, \mathbf{W E} \delta \boldsymbol{\Psi}_{T}\right\rangle \\
& =\left\langle\delta \mathbf{f}, \sum_{t=t_{1}}^{t_{2}} \mathbf{R}_{t}^{t_{2} *} \mathbf{W E} \delta \boldsymbol{\Psi}_{T} \Delta t\right\rangle .
\end{aligned}
$$

The sum is over individual time steps $\Delta t$ of the model. Given the equivalence $\delta I=\left\langle\delta \mathbf{f}, \mathbf{f}^{*}\right\rangle \Leftrightarrow \mathbf{f}^{*}=\nabla_{\mathbf{f}} I$, the expression $\mathbf{f}^{*}=\sum_{t=t_{1}}^{t_{2}} \mathbf{R}_{t}^{t_{2} *} \mathbf{W E} \delta \boldsymbol{\Psi}_{T} \Delta t$ in the scalar product of Eq. (8) is just the gradient of I with respect to a constant forcing or, for brevity, forcing sensitivity (cf. Barkmeijer et al. 2003). It has the unit of $[I] /[\mathbf{f}]$. Maps of forcing sensitivity $\mathbf{f}^{*}$ show at each point how strong an index perturbation $\delta I$ would result from a unit perturbation of the forcing of the respective state variable at that point in a forward run of the linearized model. Eq. (9) below shows how to use $\mathbf{f} *$ to construct a forcing which uses minimal energy to produce a given index perturbation $\delta I$. In a linear context this is equivalent to the maximum response at a given forcing energy. Such a forcing perturbation is termed optimal forcing in the remainder of the article. Mathematically, for a forcing perturbation $\delta \mathbf{f}$ to be optimal we require that for a given index change $\delta I=\left\langle\delta \mathbf{f}, \mathbf{f}^{*}\right\rangle$ and lead time $t_{2}-t_{1}$, the forcing perturbation has minimal "energy" $\langle\delta \mathbf{f}, \mathbf{W E} \delta \mathbf{f}\rangle$. Using the method of Lagrange multipliers yields

$$
\delta \mathbf{f}_{\mathrm{opt}}=\lambda \mathbf{E}^{-1} \mathbf{W}^{-1} \mathbf{f}^{*}, \text { with } \lambda=\frac{\delta I}{\left\langle\mathbf{f}^{*}, \mathbf{E}^{-1} \mathbf{W}^{-1} \mathbf{f}^{*}\right\rangle} .
$$

In section 3 we discuss results when we have an ensemble of trajectories taken from either the NCEPNCAR reanalysis data or from an unperturbed forward run with the model of Hall (2000). When using an ensemble of trajectories, the optimal forcing perturbation is computed using Eq. (9) applied to the average of the sensitivities, but with the amplitude rescaled so that the forcing perturbation has the same total energy norm [Eq. (6)] as the optimal forcing perturbation in the case linearized about winter climatology. We note that by averaging over the sensitivities we hope to capture some information about the eddying, nonlinear character of the real atmosphere hopefully leading to a more realistic derived forcing perturbation than for the cases that use a model linearized about the winter mean climatology.

\section{Results}

In what follows, the target or trend pattern is the total change over $51 \mathrm{yr}$ related to the linear trend during winter, 1948/49-1998/99, at each grid point and for each model variable, except that we restrict the target to the region north of $20^{\circ} \mathrm{N}$ in order to focus on the Northern Hemisphere. Figure 1 shows the trend, as it appears in Z500, and Fig. 2 shows the area-weighted (Euclidean) projection of daily mean NCEP-NCAR Z500 data on the trend pattern. Figure 2 gives an indication of the day-to-day variability in the occupation of the trend pattern by the atmosphere over all 51 winters. There is a clear trend over the 51-yr period toward more days with a stronger-than-average projection on this pattern, as expected.

\section{a. The forcing sensitivities}

We begin by using the adjoint to determine the optimal forcing perturbations for a fixed projection on the target pattern and different lead times. For the case linearized about climatology, we can test the "efficiency" with which the forcing at different lead times excites the target pattern by plotting the amplitude of the optimal forcing as a function of lead time. The forcings are the result of Eq. (9) with $\delta I=34.4 \mathrm{~kJ} \mathrm{~m}^{-2}$, which is the projection of the target upon itself, $\left\langle\delta \boldsymbol{\Psi}_{T}\right.$, WE $\left.\delta \boldsymbol{\Psi}_{T}\right\rangle$. Their amplitudes are computed using the square root of the energy norm. As can be seen from Fig. 3, the amplitude initially decreases rapidly as the lead time increases, and then levels off. Furthermore, amplitude multiplied by lead time also decreases as lead time increases. These results indicate that, at least out to 14 days, the longer the lead time the more efficient the optimal forcing becomes at exciting the trend pattern. As the lead time is increased the forcing sensitivities settle down into the patterns shown in Figs. $4 \mathrm{a}-\mathrm{c}, 5 \mathrm{a}-\mathrm{c}$, and $6 \mathrm{a}-\mathrm{c}$ for which the lead time is 12 days. (Unless stated otherwise, all of the following experiments use a 12-day lead time.) We show mostly forcing sensitivities, and not the optimal forcing perturbations themselves, because the latter have a lot of small-scale structures for vorticity and divergence (cf. Figs. 4c,d; $5 c$,d; and $6 c, d$ and see the appendix for a detailed explanation). In essence, the presence of the small-scale structures in the optimal forcing for vorticity and divergence are a consequence of using the energy norm and the need, as a consequence, to convert from vorticity and divergence to velocity. The result is that the optimal forcings for vorticity and divergence are related to their corresponding forcing sensitivities by a Laplacian operator, which in turn puts weight on small spatial scales. We note that the optimal vorticity forcing con- 


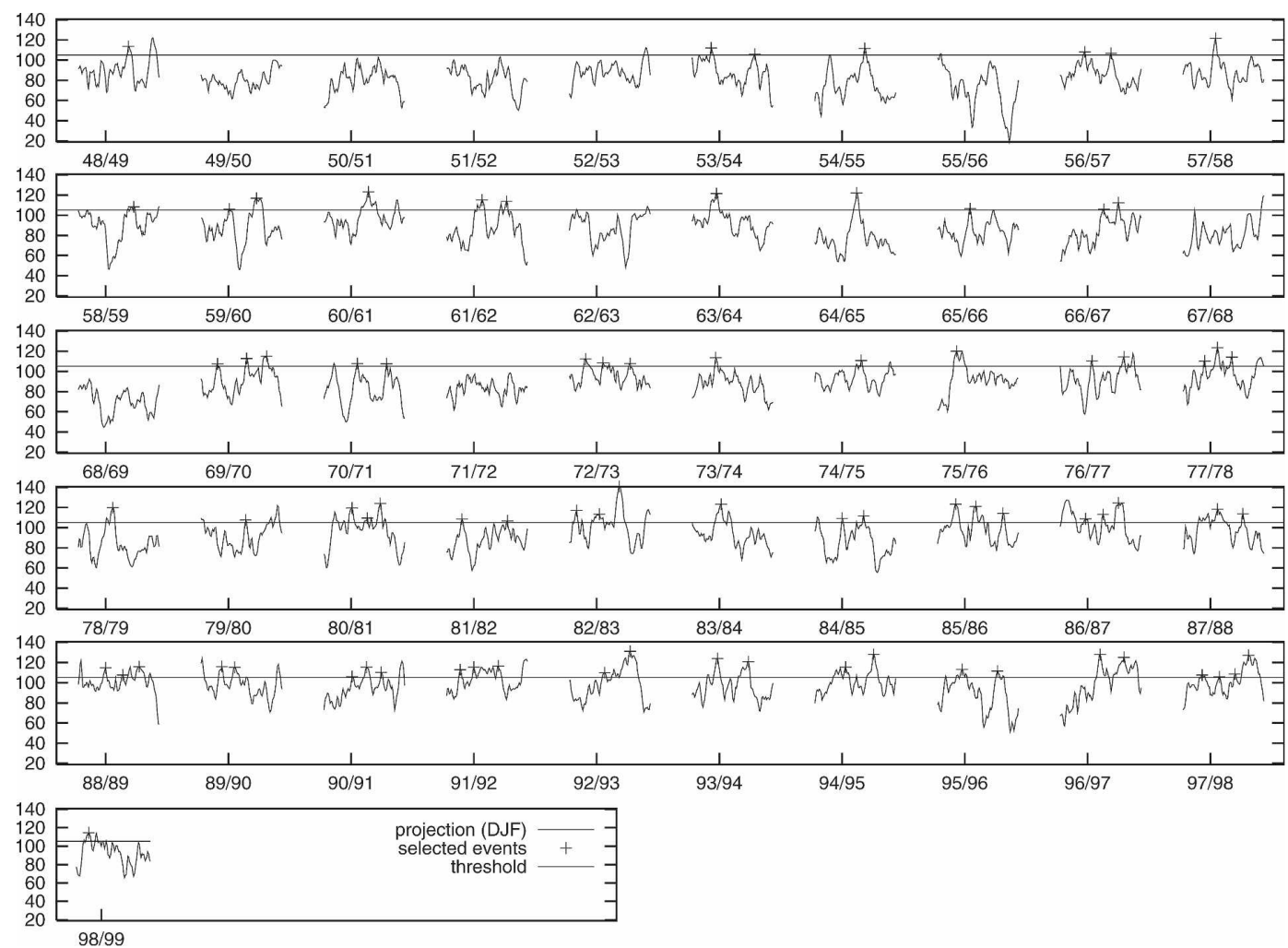

FIG. 2. Projection of daily NCEP-NCAR 500-hPa height data onto the 500-hPa trend north of $20^{\circ} \mathrm{N}$ (Fig. 1). The line separates the higher $10 \%$ from the lower $90 \%$. Crosses mark the maxima used for the subset of composites that terminate with a strong projection on the trend pattern (see text).

verted to streamfunction would have the same spatial structure as the sensitivity to vorticity forcing.

We begin by using the adjoint for the model linearized about the winter climatological mean state (Figs.

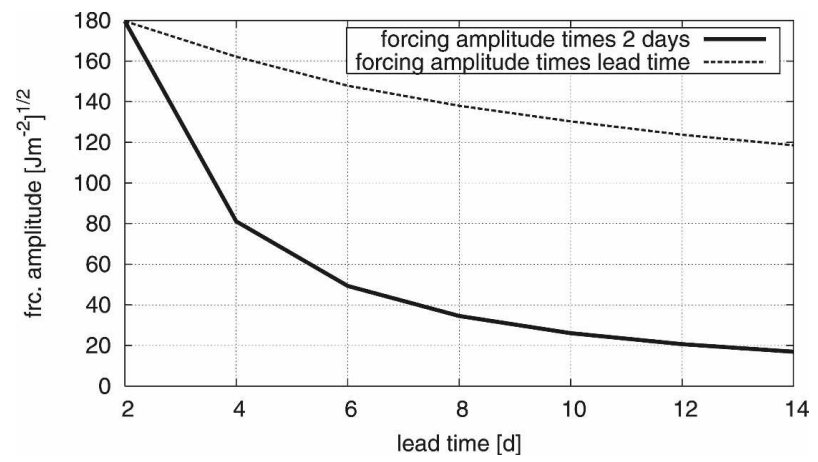

FIG. 3. Comparison of the amplitudes of the optimal forcing (solid line) and amplitude multiplied by lead time (dashed line) for different lead times of the adjoint for the case linearized about climatology. All other figures (except for Fig. 9b) use 12 days lead time. Amplitude is measured using the energy norm. For ease of plotting, the amplitude of the forcing in the solid line case is multiplied by 2 days. 4a, 5a, and 6a) and then go on to consider the adjoint for a tangent linear model linearized about timeevolving states (Figs. 4b,c; 5b,c; and 6b,c). Results from areas where the flat bottom of the model is in strong contradiction with reality, namely the Himalaya, are omitted. Looking first at the vorticity sensitivity for level 3 (at about $500 \mathrm{hPa}$ ) shown in Fig. 4a, we see what looks like a Rossby wave train originating over the tropical western Pacific south of our cutoff latitude of $20^{\circ} \mathrm{N}$. This feature is present at other model levels and has corresponding features in the forcing sensitivities for other variables. It is reminiscent of a Rossby wave source (e.g., Sardeshmukh and Hoskins 1988) and is in a similar (though not identical) position to the Rossby wave source identified by Greatbatch and Jung (2007) as being effective in forcing the positive NAO in the ECMWF model. Nevertheless, it is important to realize that what is plotted is forcing sensitivity, and so is not actually a Rossby wave itself. Rather, the adjoint technique selects the optimal forcing that excites a model response with a given projection onto the trend pattern. It follows that if a Rossby wave train emitted from the tropical Pacific is a potentially important part of the 


\section{(a) climate sensitivity}

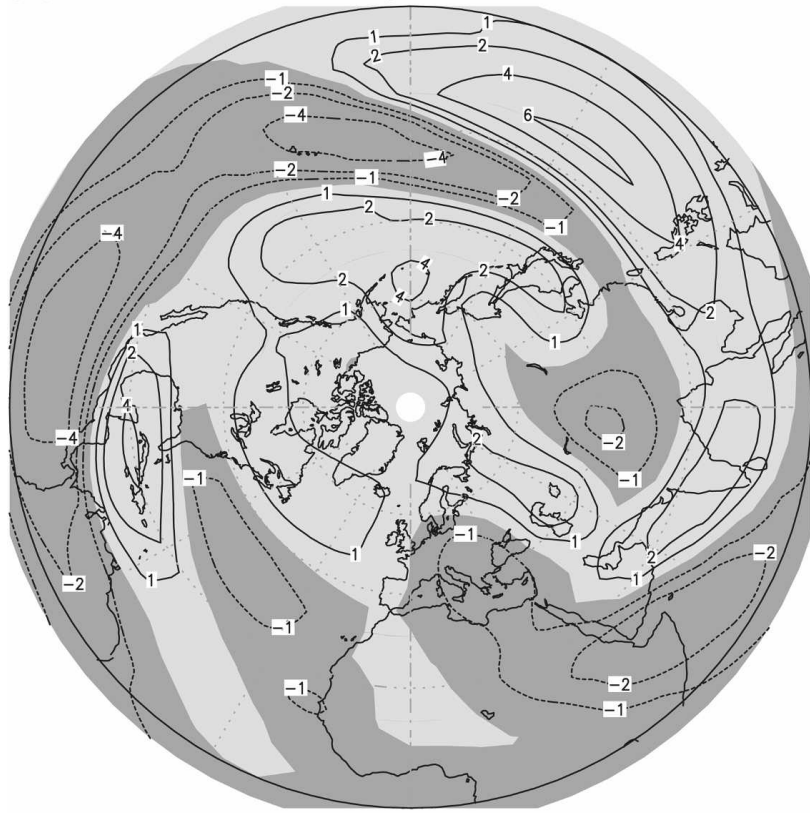

(c) PUMA ensemble mean sensitivity

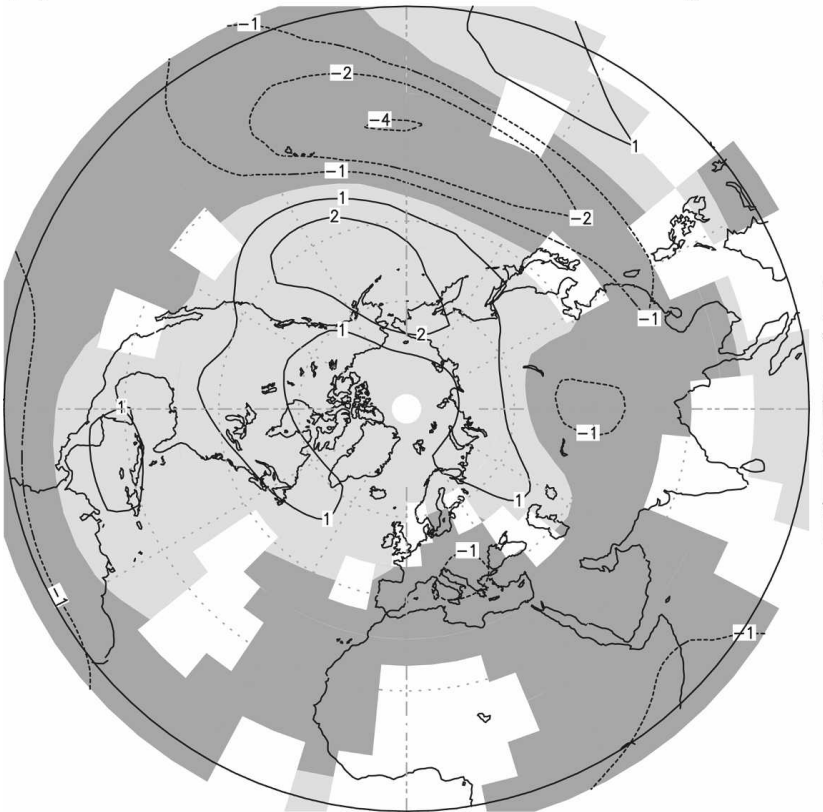

(b) NCEP ensemble mean sensitivity

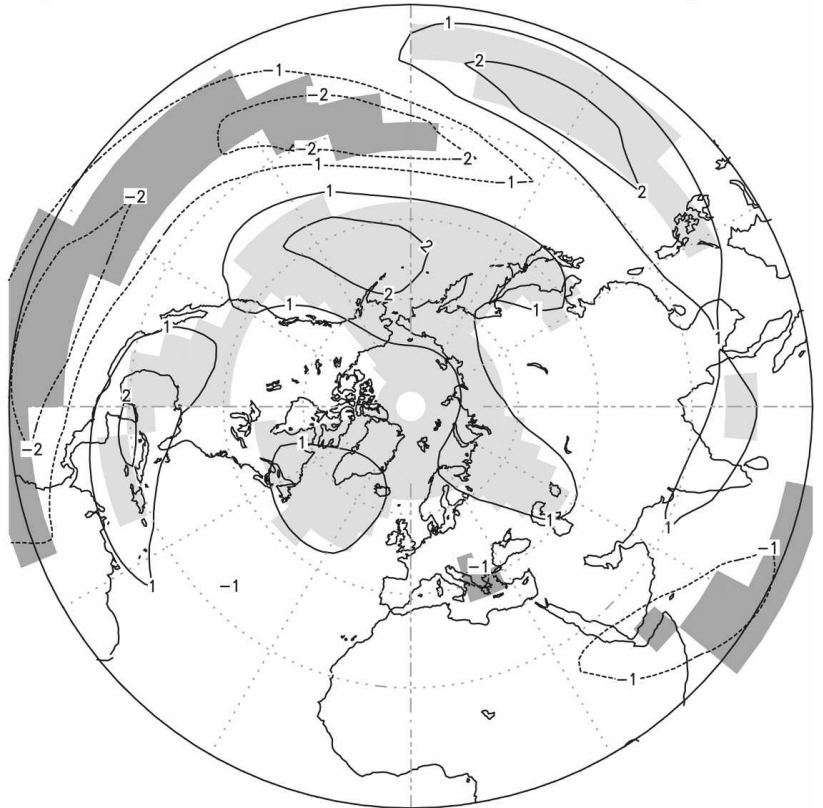

(d) PUMA forcing perturbation

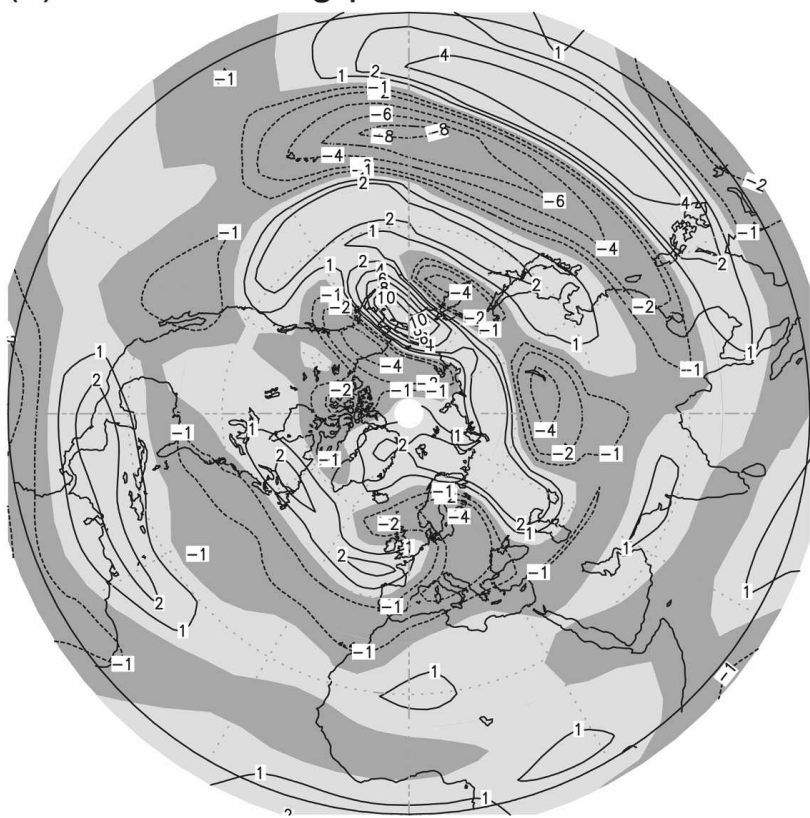

FIG. 4. Sensitivities $\left(\times 10^{12} \mathrm{Jm}^{-2} \mathrm{~s}^{-1}\right)$ for the vorticity forcing in the middle model level (about $500 \mathrm{hPa}$ ), showing at each point how much the projection on the trend pattern would change in a linear 12-day integration if at this point a forcing of unit amplitude was applied. From (a) the linear adjoint experiment, (b) averaged over many realizations of the adjoint using the NCEP-NCAR trajectories, and (c) averaged over many realizations of the adjoint PUMA experiment. (d) Optimal forcing perturbations $\left(\times 10^{-12} \mathrm{~s}^{-2}\right)$ derived from (c). In (b) and (c) only sensitivities that are significant on the $10 \%$ level are shaded (light gray: positive; dark gray: negative). All sensitivities are scaled to relate to the full observed amplitude of the trend pattern. Optimal forcing perturbations in (d) are scaled to have the same global amplitude as in the case linearized about climatology (see text for details).

trend, then the adjoint approach will find the most efficient way to excite the Rossby wave train within the given lead time, and this may not be the same way that the Rossby wave train was excited in nature. For ex- ample, directly forcing the pattern of the Rossby wave train itself (as appears to be the case here) could well be more efficient than a diabatic heat source in the tropical Pacific, even if in reality the latter were the physically 
(a) climate sensitivity

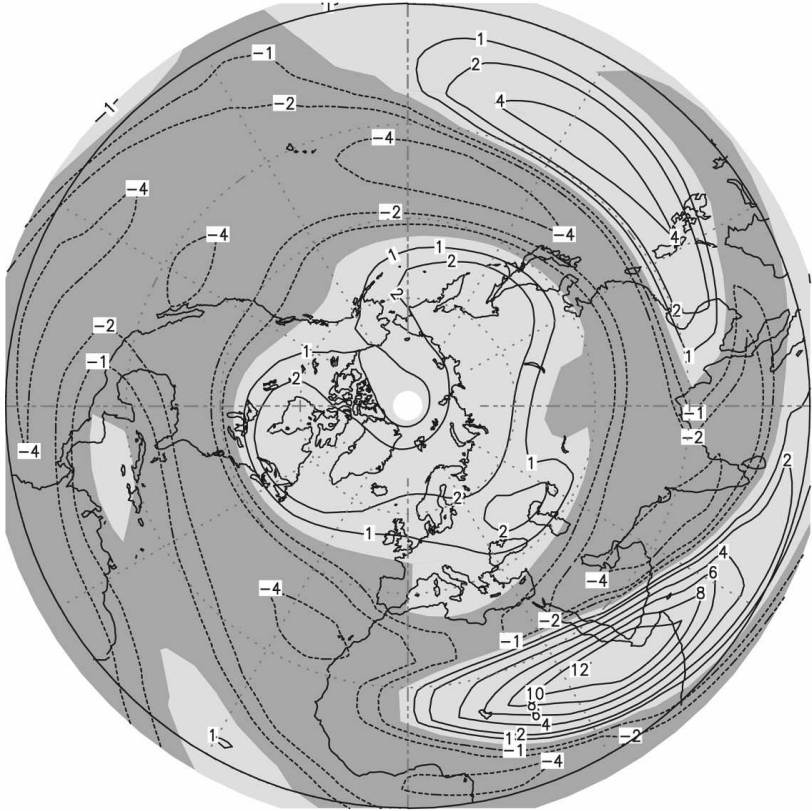

(c) PUMA ensemble mean sensitivity

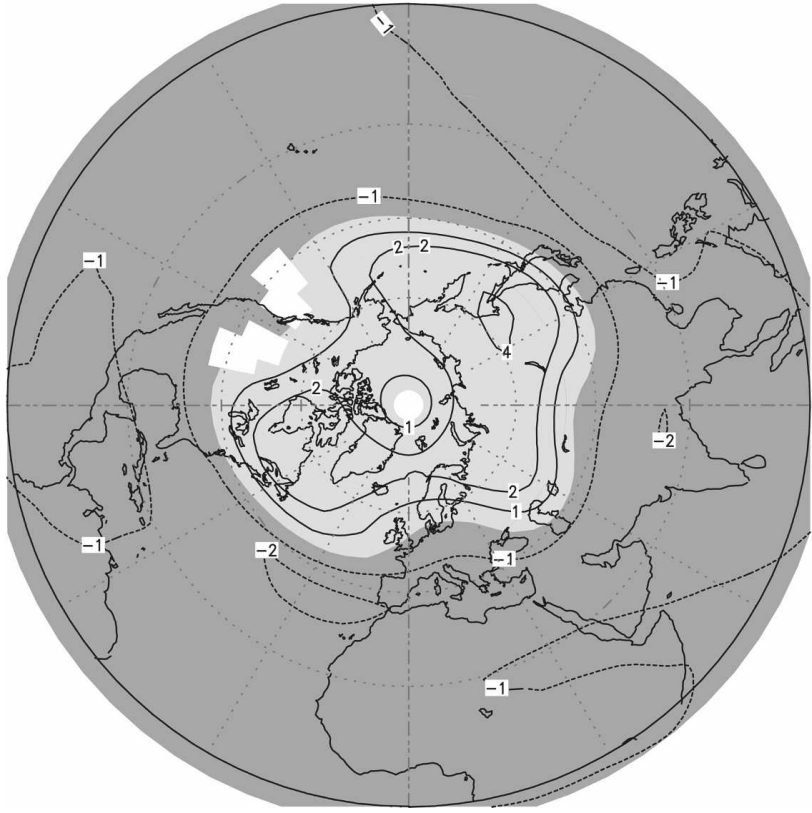

(b) NCEP ensemble mean sensitivity

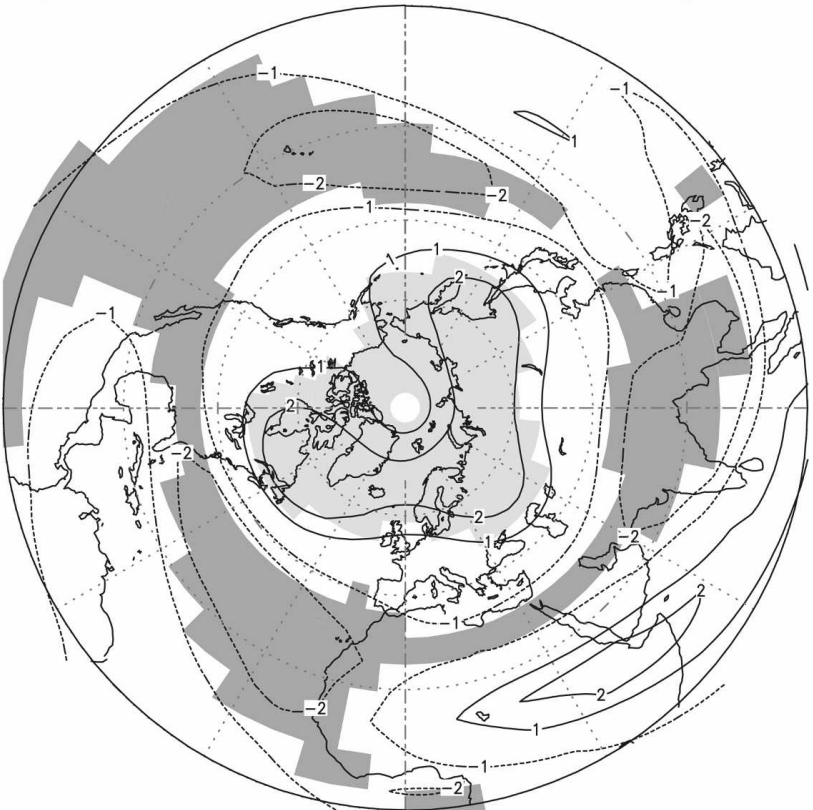

(d) PUMA forcing perturbation

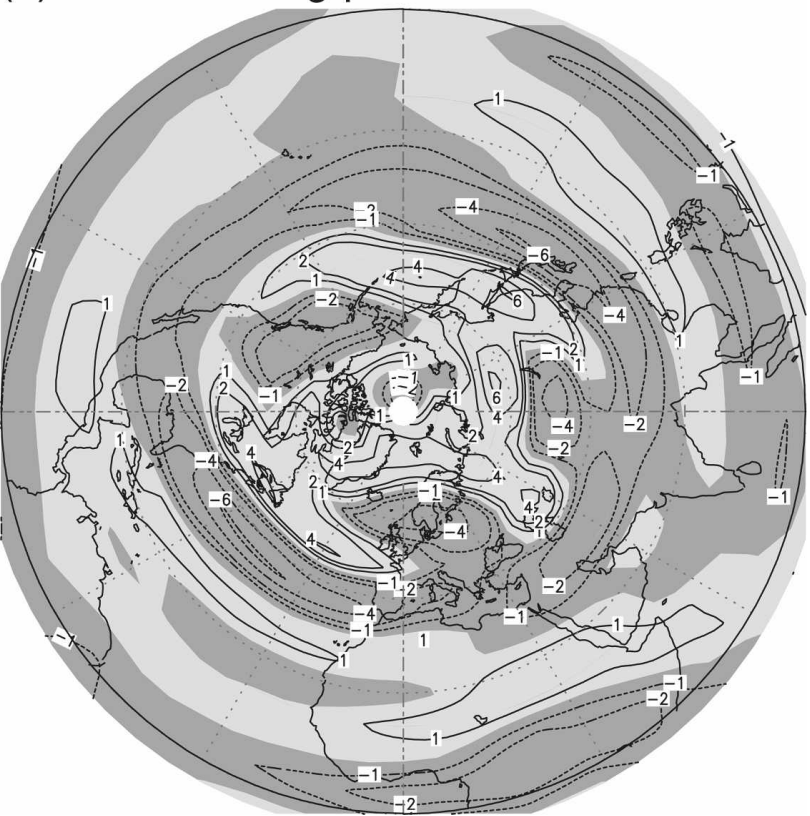

FIG. 5. As in Fig. 4 but for vorticity forcing in the highest model level (about $100 \mathrm{hPa}$ ).

relevant forcing perturbation. It should also be noted that the way the model finds it most efficient to excite a particular feature probably also depends on the resolution of the model.

Nevertheless, a Rossby wave source in this region is broadly consistent with the finding of Lu et al. (2004) that forcing from the tropical Indo-Pacific region is important for driving the target pattern (i.e., the 51-yr trend). The importance of forcing over the western tropical Pacific for driving the trend has also been noted by Kucharski et al. (2006) in forward model runs using a simplified GCM.

In addition to the Rossby wave feature, Fig. 4a also exhibits an annular pattern with implied positive vorticity forcing over the polar region and implied forcing of the opposite sign around the $30^{\circ} \mathrm{N}$ latitude belt, in- 
(a) climate sensitivity

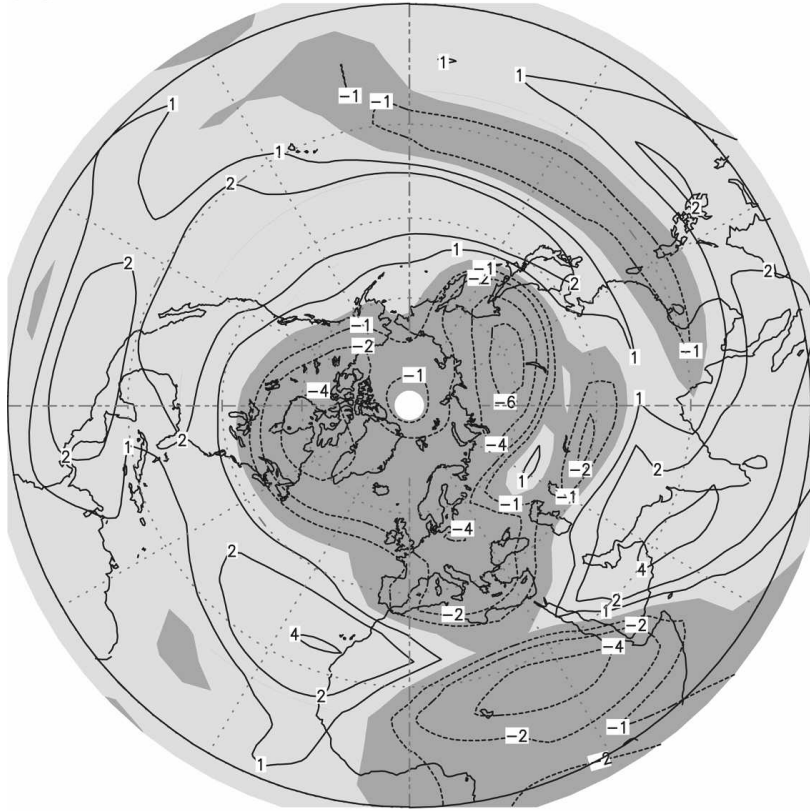

(c) PUMA ensemble mean sensitivity

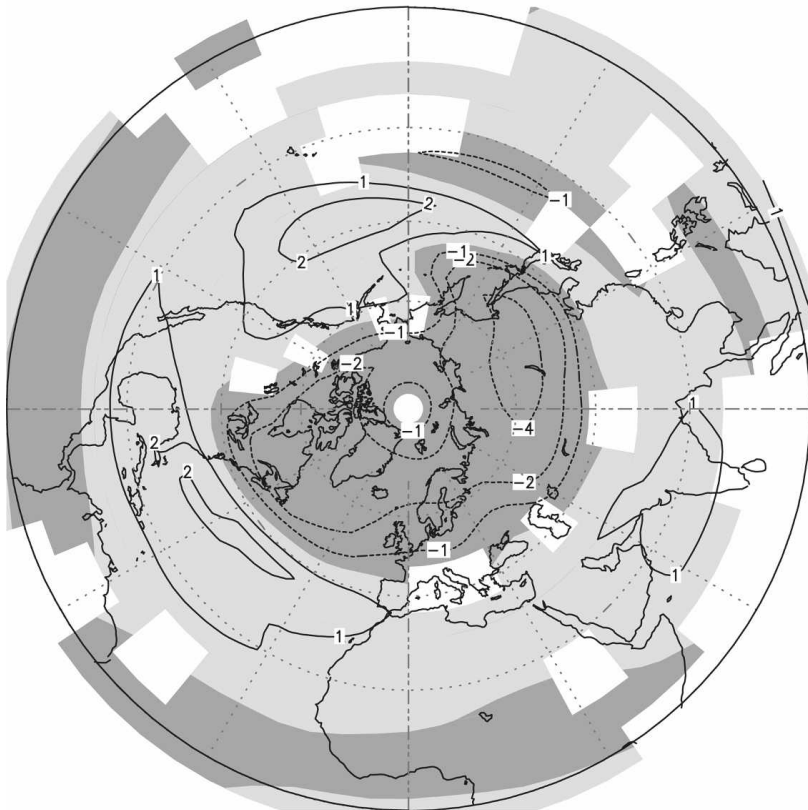

(b) NCEP ensemble mean sensitivity

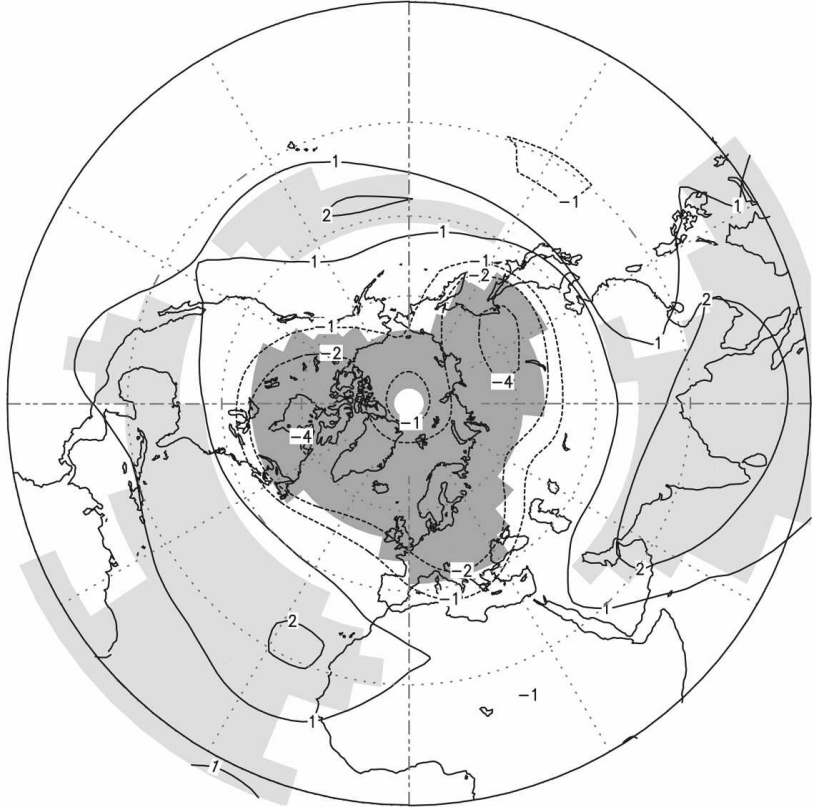

(d) PUMA forcing perturbation

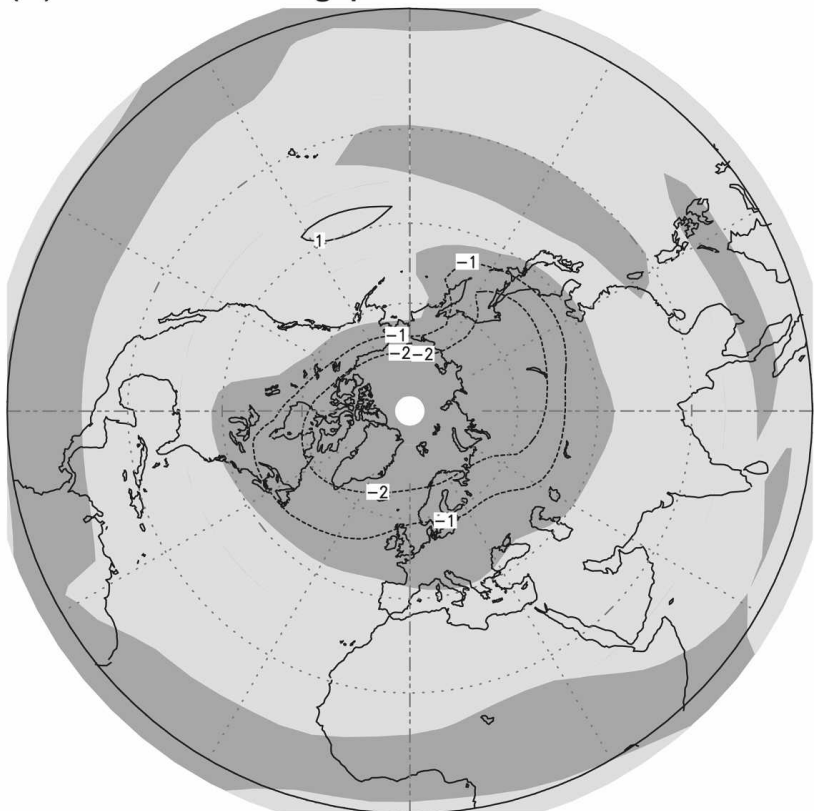

FIG. 6. As in Fig. 4 but for temperature forcing in the highest model level $(100 \mathrm{hPa})$. (a)-(c) Sensitivities $\left(\times 10^{6} \mathrm{~J} \mathrm{~m}^{-2} \mathrm{~K}^{-1}\right)$ and $(\mathrm{d})$ optimal perturbations $\left(\times 10^{-6} \mathrm{Ks}^{-1}\right)$.

dicative of forcing for the positive phase of the northern annular mode (or Arctic Oscillation; Thompson and Wallace 2000). The annular forcing feature is even clearer in the top-model-level vorticity-forcing sensitivity (Fig. 5a), although the Rossby wave feature can be seen here too. Looking at the sensitivity for temperature in the top model level (Fig. 6a), we again see both the annular mode-type forcing pattern, with cooling over the pole and warming at lower latitudes, and the Rossby wave-type pattern originating from the tropical western Pacific. It is possible that the presence of the annular mode-type forcing in the uppermost model levels mimics influences from the stratosphere. Such a view is consistent with the work of Scaife et al. (2005), 
who have argued an important role for the stratosphere in explaining the trend. Other features in Figs. 4a, 5a, and $6 \mathrm{a}$, such as the large forcing anomaly over east Africa in Fig. 5a, become less important when timeevolving trajectories are considered, as we discuss next.

Next we turn to forcings derived using the tangent linear model linearized about time-evolving trajectories. We begin by using trajectories obtained by projecting daily mean realizations from the NCEP-NCAR reanalysis onto the model grid. Each 12-day integration using the adjoint model yields sensitivity fields, which are then averaged to produce a "composite" sensitivity to forcing, and this "composite" sensitivity is then tested for significance against the null hypothesis of zero sensitivity (for a discussion on how the corresponding forcing perturbation is derived from average of the sensitivities, see section 2). Here we use 1000 partly overlapping trajectories, each using a different daily realization as the initial condition. The results are shown in Figs. 4b, 5b, and 6b. We have also considered a subset of trajectories selected on the basis that the trajectories terminate with a strong projection on the target pattern (see Fig. 2). The results for this subset are basically the same as for the case using all available trajectories and are not discussed further here. In the figures, regions where the sensitivity is significantly different from zero at the $10 \%$ level are shaded (light gray: positive; dark gray: negative). It is immediately clear that the main features in Figs. $4 b, 5 b$, and $6 b$ are the same as in Figs. 4a, 5a, and 6a, indicating that linear dynamics, linearized about the winter climatological mean state, is an important aspect of the dynamics of the 51-yr trend in this model, consistent with $\mathrm{Lu}$ et al. (2004). We can investigate this further using trajectories taken from a fully nonlinear, time-evolving model run, rather than based on observations. Here, the model run uses time-independent climatological forcing, as in Hall (2000), and corresponds to the same model setup that forms the basis for our adjoint model. Again, 1000 trajectories are used. The results are shown in Figs. $4 c, 5 c$, and $6 c$, together with the corresponding optimal forcing perturbation in Figs. 4d, 5d, and 6d (the amplitude of the latter being determined as for the case linearized about NCEP-NCAR trajectories). The sensitivities are again found to be very similar to those shown in Figs. 4a,b; 5a,b; and 6a,b, except that, compared to the cases linearized about NCEP-NCAR trajectories (Figs. 4b, 5b, and 6b), there is an increase in the area where the forcing sensitivity is significantly different from zero, reflecting the reduced spread of the trajectories taken from the model compared to those based on observations. It is interesting that, when linearizing about time-evolving trajectories, the Rossby wave-type feature from the tropical Pacific is reduced in amplitude compared to the annular forcing pattern in the top model level (Fig. 5), especially when using model trajectories (Figs. 4c, 5c, and 6c). In addition, in Fig. 4 the part of the Rossby wave feature that extends westward over the Asian continent, as well as the feature over Africa in the case linearized about climatology (Fig. 4a), is also reduced in importance when using time-evolving trajectories (Figs. 4b,c).

Finally in this section we note that the amplitude of the optimal forcings we have derived are not unrealistic. For example, the temperature forcing shown in Fig. 6d corresponds to a forcing at $100 \mathrm{hPa}$ of less than 0.2 $\mathrm{K}_{\text {day }}{ }^{-1}$ and the vorticity forcing in Fig. $4 \mathrm{~d}$ to $\approx 10^{-5} \mathrm{~s}^{-1}$ $\mathrm{day}^{-1}$ at the maximum.

\section{b. Forward runs using a linear model}

We now verify the optimal forcings derived above by using them to drive a model linearized about the climatological winter mean flow. The model is based on the PUMA code and uses the same dissipation parameters as in the fully nonlinear model of Hall (2000). In each case, the model forcing is that derived with a lead time of 12 days, and the model, in turn, is run forward for 12 days. Figure $7 \mathrm{~b}$ shows the response pattern to the forcing diagnosed from the model linearized about climatology. It should be noted that the amplitude of the forcing used to produce Fig. $7 \mathrm{~b}$ is determined from Eq. (9) with $\delta I$ equal to the projection (using the energy norm) of the target on itself (this choice is made so that the amplitude of the model response can be compared with Fig. 1, repeated for convenience as Fig. 7a). If we define correlation and amplitude using the energy norm, the model response has a correlation of 0.46 with the target pattern but a larger amplitude by a factor of 2.2. By construction, for this case the projection onto the target is $100 \%$, corresponding to the projection of the target onto itself. The main centers of action are reproduced, but with some shortcomings over Asia and southern North America and with the amplitude of the Aleutian low overemphasized. The model does not reproduce the target pattern exactly because the "optimality" of the derived forcing perturbation is measured in terms of projection onto the target using the energy norm and does not require that the model response correspond exactly to the target itself. Chiefly the result is a mathematical compromise between the growth rate of internal modes and their respective pattern correlation with the target. Figure $7 \mathrm{c}$ shows the model response to the forcing derived from the average of the sensitivities obtained using trajectories derived from the NCEP-NCAR reanalysis. The amplitude of the forcing in this case is chosen to be the same as the 
(a) NCEP Z500 trend

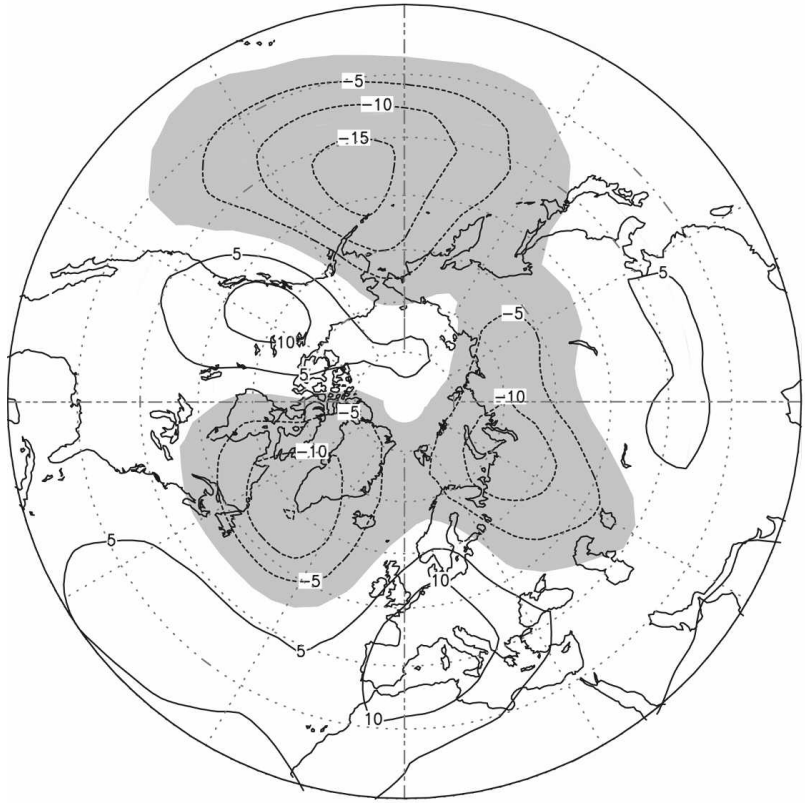

(c) response to NCEP forcing

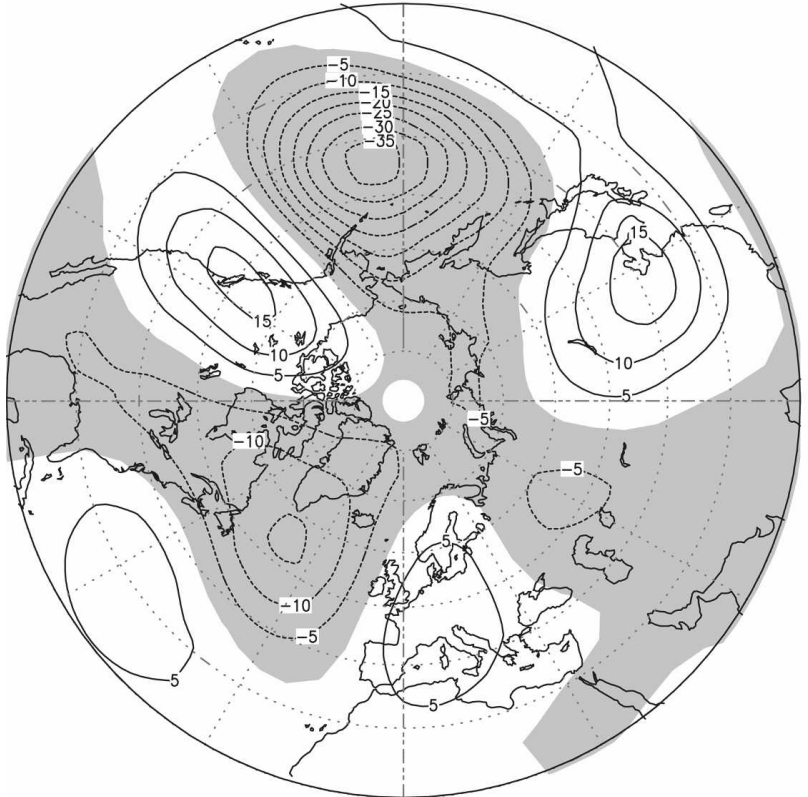

(b) response to clim. forcing

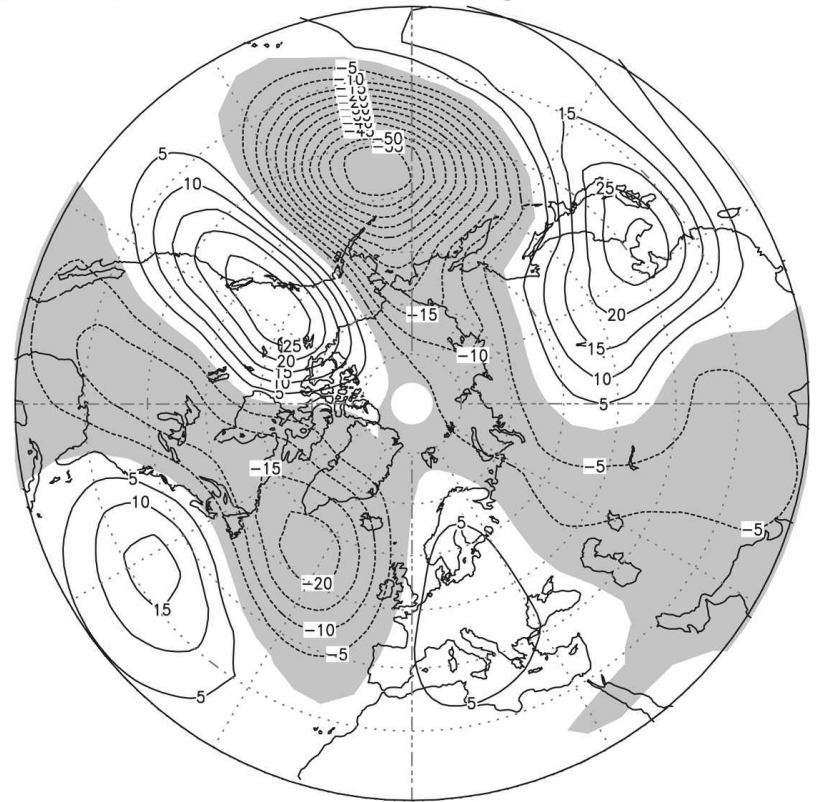

(d) response to PUMA forcing

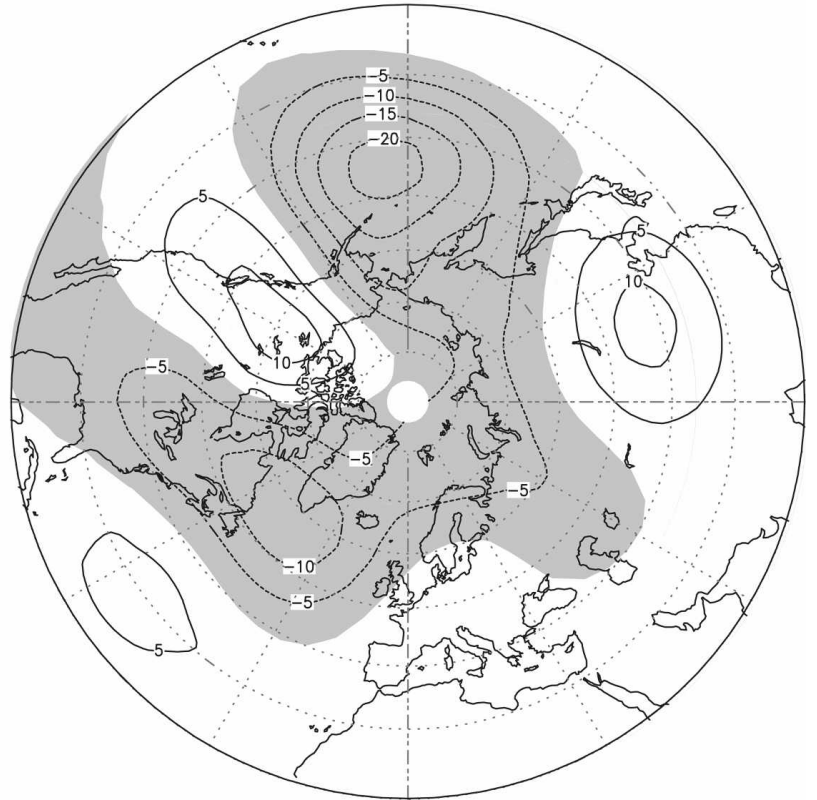

FIG. 7. (a) Linear trend in 500-hPa height from Fig. 1, and (b) 500-hPa response in a 12-day run linearized about NCEP-NCAR climatology to the optimal anomalous forcing. The forcing is scaled to produce the same projection as the trend projected onto itself $\left(34.4 \mathrm{~kJ} \mathrm{~m}^{-2}=100 \%\right)$. The amplitude of the response is 2.2 times larger than the target pattern, and the pattern correlation is 0.46 . (c) The same for the anomalous forcing derived from averaged sensitivities using the NCEP-NCAR trajectory. With the forcing scaled as in the optimal case the response reaches a relative amplitude of 1.4 , the projection on the target is $73 \%$, and the pattern correlation is 0.55 . (d) The same for the anomalous forcing derived from averaged sensitivities using a PUMA trajectory. With the forcing scaled as in the optimal case, the response reaches a relative amplitude of 0.91 , the projection on the target is $54 \%$, and the pattern correlation is 0.59 . Contours are every $5 \mathrm{~m}(10 \mathrm{yr})^{-1}$ and negative regions are shaded.

amplitude of the forcing used to produce Fig. 7b. This time the amplitude of the model response is weaker (only 1.4 times that of the target amplitude), but the correlation is increased to 0.55 . On the other hand, the projection on the target is $73 \%$ of the projection of the target on itself. The reduced projection is an indication that the forcing is less efficient at exciting the target than the previous forcing for the particular model being 
(a) ens. mean response, pos. forcing

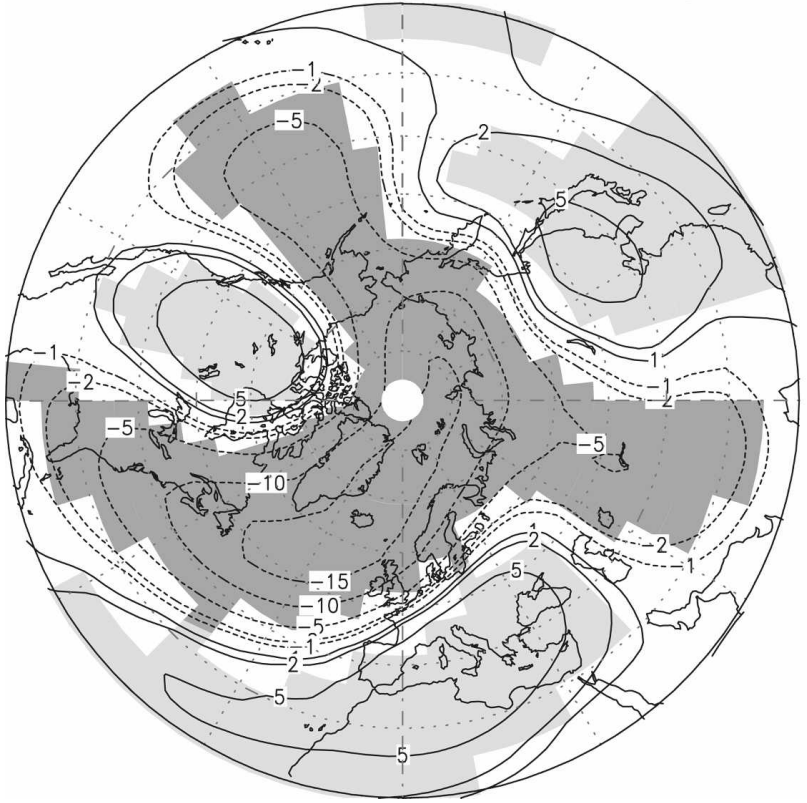

(b) ens. mean response, neg. forcing

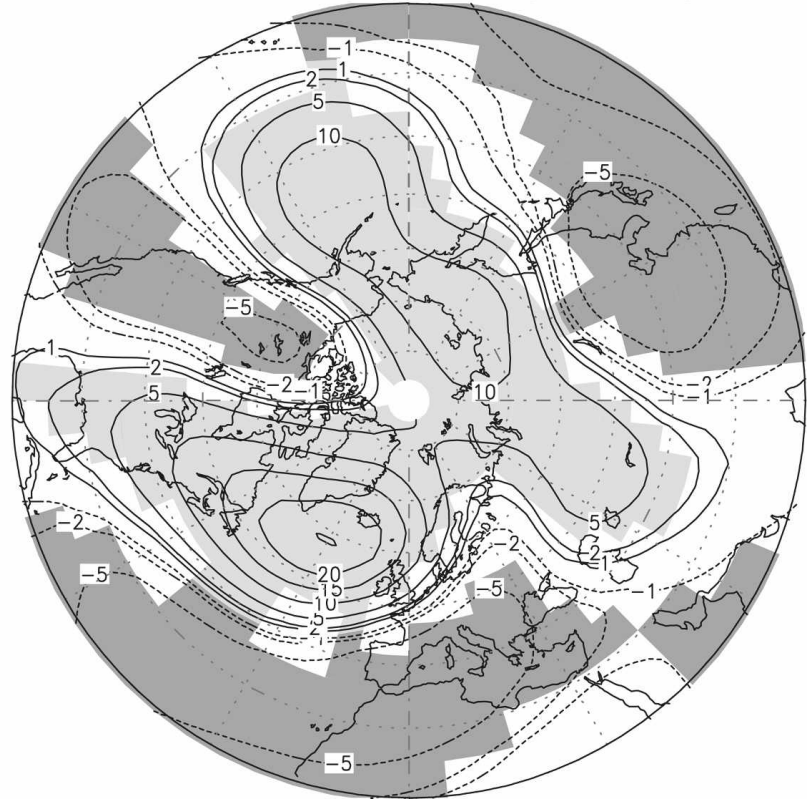

FIG. 8. Nonlinear ensemble mean response to forcing derived using a PUMA trajectory. Shown is 500-hPa geopotential height difference from a control ensemble, significant on the $10 \%$ level. The forcing was scaled to yield the same projection in a linear run as the full observed trend, and the result was divided by the number of decades to make it comparable to Fig. 1. (a) Positive and (b) negative forcing. Contours are at 1,2, and then every $5 \mathrm{~m}$, significant positive (negative) areas are shaded in light (dark) gray.

used here (that is a model linearized about climatology). We have also used forcing derived from the timeevolving model trajectories (see Fig. 7d), where the amplitude of the forcing is again the same as the amplitude of the forcing used to produce Fig. $7 \mathrm{~b}$. In this case, the model response has even less amplitude (0.91), a slightly higher correlation (0.59), but an even smaller projection on the target of $54 \%$ of the projection of the target on itself, indicating that the forcing in this case is even less efficient at exciting the target pattern for the particular model used here (i.e., linearized about climatology).

\section{c. Forward runs using a nonlinear model}

We now turn to forward model runs that use the fully nonlinear dynamical model, in this case the model of Hall (2000), except that the model core uses the PUMA code. The model forcing is the climatological mean forcing used by Hall (2000), with the climatological forcing perturbed by the forcing derived using the adjoint model. For the model runs shown here, different perturbed forcings are considered using different amplitudes and derived using different lead times. In all cases, the perturbed forcing is derived from the tangent linear model applied to model trajectories, as in Fig. 7d described above, as this is the forcing considered most consistent with the model dynamics. For each particular choice of forcing, an ensemble of 30 model runs is created, each ensemble member being initialized with a random realization from the NCEP-NCAR reanalysis projected onto the model grid and run for 6 months with only the last 3 months being used for analysis. For comparison with the perturbed runs, a control run is also carried out using climatological forcing only (the perturbed forcing is zero), this time using 100 ensemble members. Figure 8 shows the ensemble mean model response to the perturbed forcing (perturbed forcing minus control) when the amplitude in terms of the total energy norm of the perturbed forcing is the same as used to produce Fig. 7d. Positive and negative forcing cases are shown. The same basic structure is seen when the amplitude is reduced to $1 / 3$ of that used here, or if forcing derived for a lead time of 6 days is used, rather than the 12-day lead time used here. We see that the ensemble mean model response is quite similar (but with the sign reversed in the negative forcing case) to both that of the target pattern (Fig. 7a) and of that produced by the linear model (Fig. 7d), indicating that forcing derived using an adjoint with a lead time of days (in this case 12 days) can systematically excite a pattern similar to that of the target when applied in a nonlinear dynamical model. Compared to Fig. 7d, the model response in the nonlinear case shows a weakening of the 
(a) 12 days lead

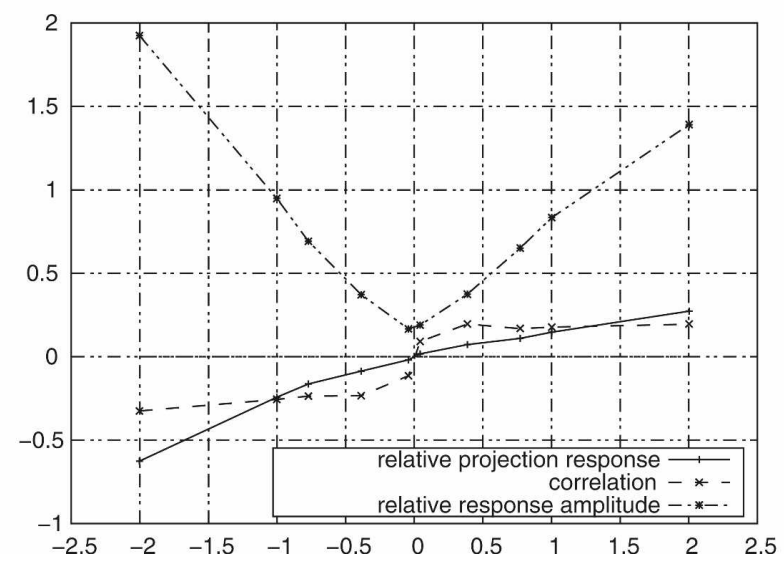

(b) 6 days lead

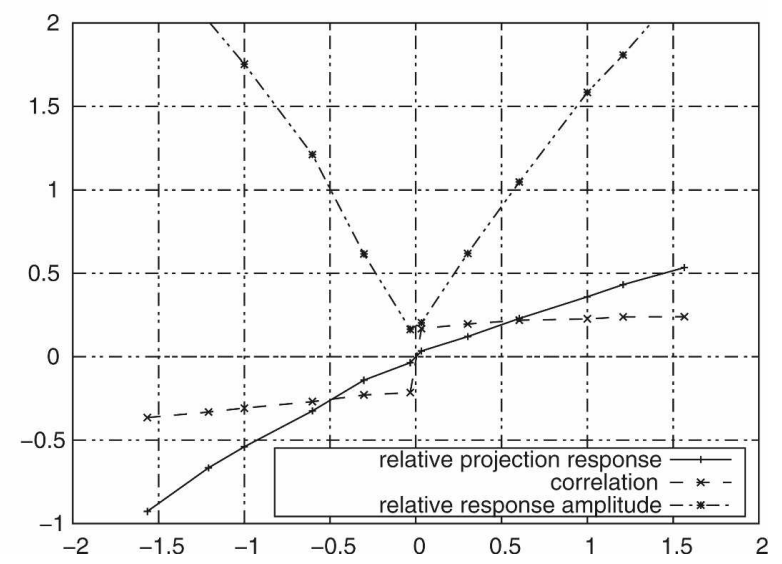

FIG. 9. Ensemble mean responses in nonlinear runs to anomalous forcings optimal for lead times of (a) 12 and (b) 6 days. The $x$ axes give the amplitude of the applied forcing relative to the respective optimal forcing in the fully linear experiment. The $y$ axes are scaled with the projection of the full trend pattern onto itself (except for correlation). We show projection onto the trend pattern (solid line), correlation with the trend pattern (dashed), and amplitude of the response measured in the energy norm (dotted). The first quantity is the product of the latter two.

Aleutian low and a deepening of the Icelandic low features. Figures 9a,b show plots of amplitude, correlation, and projection (using the energy norm and where projection is relative to that of the projection of the target pattern on itself) for different amplitudes of the forcing, where 1 on the abscissa corresponds to the forcing amplitude used in the linear model run shown in Fig. 7b. The fact that the correlations are roughly constant either side of zero indicates the model response is rather linear with increasing amplitude of the forcing. We also see that the amplitude of the model response also increases with an increase of the forcing amplitude, although this increase is steeper for the negative forcing cases. Likewise, the projection suggests essentially linear behavior. Using forcing derived with a lead time of 6 days gives similar results (see Fig. 9 b).

As noted in the introduction, previous studies (e.g., Corti and Palmer 1997; Jung and Barkmeijer 2006) have shown that nonlinear models, perturbed by forcing derived using an adjoint model with a lead time of days, show a systematic tendency to excite the corresponding target pattern. Our results confirm this effect. We suggest that the connection between forcing derived using lead times of, say, 10 days, and forcing that systematically excites a pattern in an ensemble mean sense on climate time scales, arises because the dissipation time scale in the atmosphere is typically measured in tens of days, a time scale comparable to the lead times we have considered. Nevertheless, a close comparison between Figs. 7d and 8a shows that, in the nonlinear runs, the anomalies associated with the Aleutian low tend to be weaker than in the linear model runs, whereas the anomalies associated with the Icelandic low tend to be increased for both positive and negative anomalous forcing (most likely because of systematic forcing from the eddies in the nonlinear runs). In addition, the features in the ensemble mean response tend to be shifted slightly eastward in their positions compared to their linear counterparts.

\section{d. Forcing restricted to parts of the model domain}

We begin by examining the role played by the Rossby wave feature noted in the forcing sensitivities (Figs. 4-6), and which originates in the western tropical Pacific region. To do this, the forcing perturbations for all model variables diagnosed using the PUMA trajectories are restricted to the western tropical Pacific (WTP; $30^{\circ} \mathrm{S}-30^{\circ} \mathrm{N}, 120^{\circ}-210^{\circ} \mathrm{E}$; see Figs. $4 \mathrm{c}, \mathrm{d}$ ). This part of the forcing contributes $18 \%$ of the energy (measured using the squared energy norm) of the total forcing in all variables. Figure 10a shows the response of $500-\mathrm{hPa}$ height to this forcing in the model linearized about winter climatology. It accounts for $12 \%$ of the projection of the response to full forcing onto the target pattern with a pattern correlation of 0.24 . As expected, the model response shows a wave train emanating from the western tropical Pacific and extending across North America, not unlike the pattern shown in $\mathrm{Lu}$ et al. (2004, their Figs. 5d,e) when their model forcing was restricted to the western tropical Indo-Pacific region, but with the centers of action slightly shifted. These results confirm the importance of the Rossby wave feature in the forcing sensitivities for exciting this part of 
(a) lin. response to WTP forcing

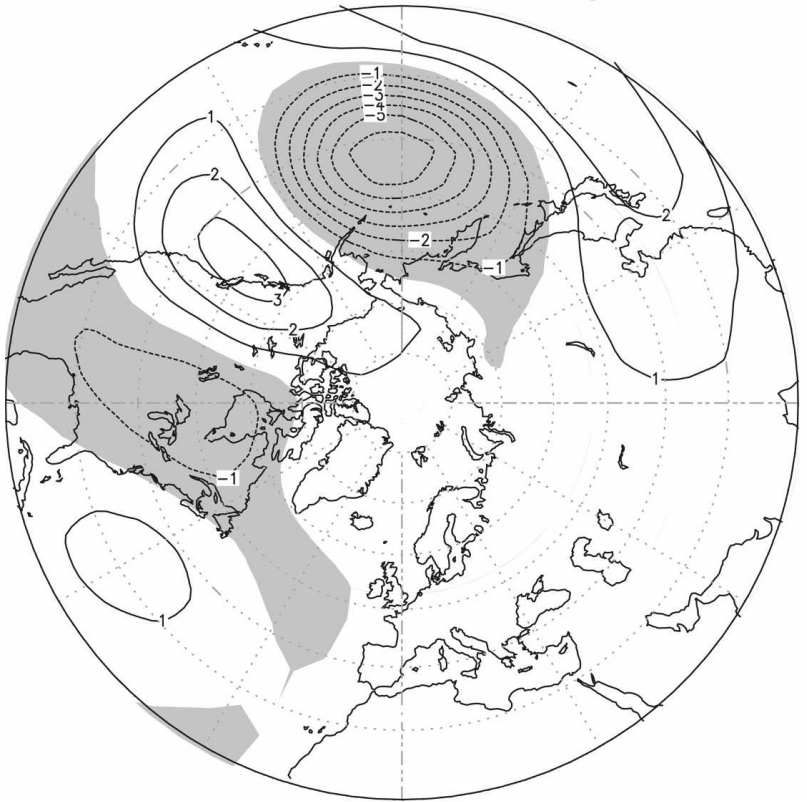

(b) lin. response to top-level forcing

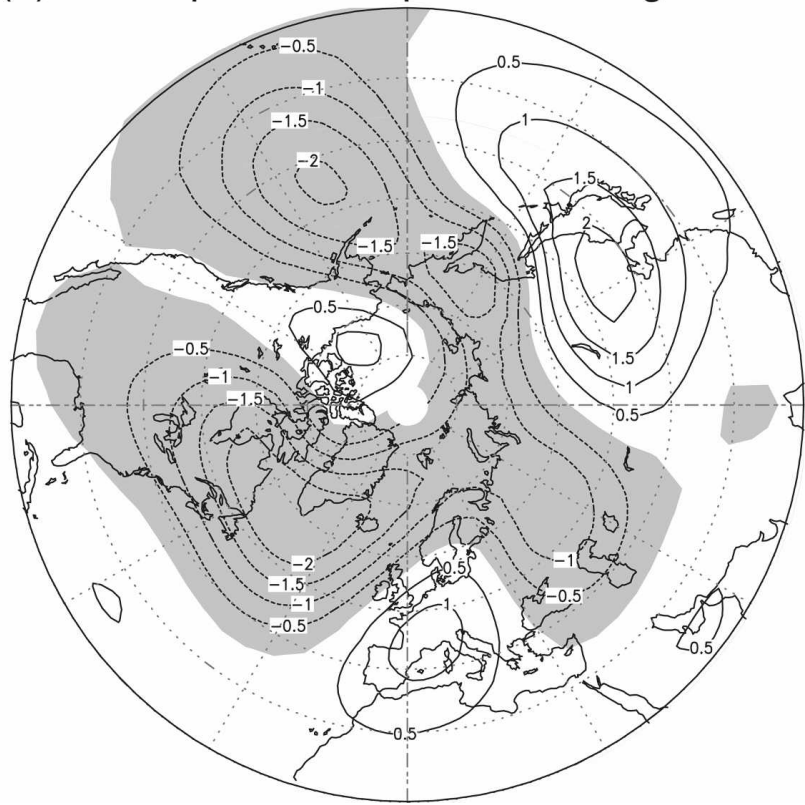

FIG. 10. As in Fig. 7d (response in linear model to forcing derived from PUMA trajectories), but with the anomalous forcing restricted to (a) the western tropical Pacific $\left(30^{\circ} \mathrm{S}-30^{\circ} \mathrm{N}\right.$ and $\left.120^{\circ}-210^{\circ} \mathrm{E}\right)$ and (b) the top model layer (about $\left.100 \mathrm{hPa}\right)$.

the trend pattern, supporting claims by a number of authors (e.g., Hoerling et al. 2004; Hurrell et al. 2004; Lu et al. 2004; Kucharski et al. 2006). We now restrict the anomalous forcing (forcing for all variables) to the top model level (about $100 \mathrm{hPa}$ ). The response of the linear model about climatology is shown in Fig. 10b. The model response accounts for $28 \%$ of the projection of the full response onto the target with a pattern correlation of 0.56 . In this case, the model response is very similar to that of the trend pattern itself, but with reduced amplitude, and captures the features of the trend pattern over the Euro-Atlantic/Asian sector not accounted for by the Rossby wave-type forcing (cf. Fig. 10a).

The importance of the forcing in the top model level for driving an annular-type response can be seen in Fig. 11a. Here the ensemble mean response of the nonlinear model (ensemble minus control, as before) is shown for the same forcing as used to produce Fig. 10b. Interestingly, the amplitude of the model response is greatly increased in this case compared to the linear model run, and is about two-thirds of the amplitude when the full anomalous forcing is used (cf. Fig. 8). This suggests that synoptic eddies in the troposphere can act to amplify signals that are imposed on the troposphere from the overlying stratosphere (albeit in the very simple model setting being used here). For the case shown, the amplification is by a factor of up to 5 compared to the linear model; indeed, the model response over the
North Atlantic in this case is comparable to that in the trend pattern itself (Fig. 1). Our results, therefore, provide some corroboration for the finding of Scaife et al. (2005) that imposing the observed trend on the stratosphere in a model can drive a trend similar to that observed in the troposphere over the Euro-Atlantic sector. We note, however, that although the model response in this case implies a deepening trend for the Icelandic low, similar to what was observed, the opposite trend is implied in the North Pacific; that is, a trend toward a less deep Aleutian low than in climatology and the opposite of what was observed. Interestingly, the spatial pattern associated with the northern annular mode also shows a weakened Aleutian low together with a deepened Icelandic low (Thompson and Wallace 2000). Clearly further studies using higher-resolution model versions (especially higher vertical resolution) will be required to explore further the role played by the stratosphere in the trend. We also show the response of the nonlinear model to the negative of the forcing used to produce Fig. 11a. In this case (Fig. 11b), the model response is similar in both pattern and amplitude to the negative of the response in Fig. 11a, suggesting that a negative trend in the stratosphere can excite a similar negative trend in the underlying troposphere. Finally, for completeness we show the result of two nonlinear model runs using both signs of the forcing used to produce Fig. 10a. In this case (Figs. 11c,d), the nonlinear model leads to some distortion of the 
(a) ens. mean resp. to top level forcing

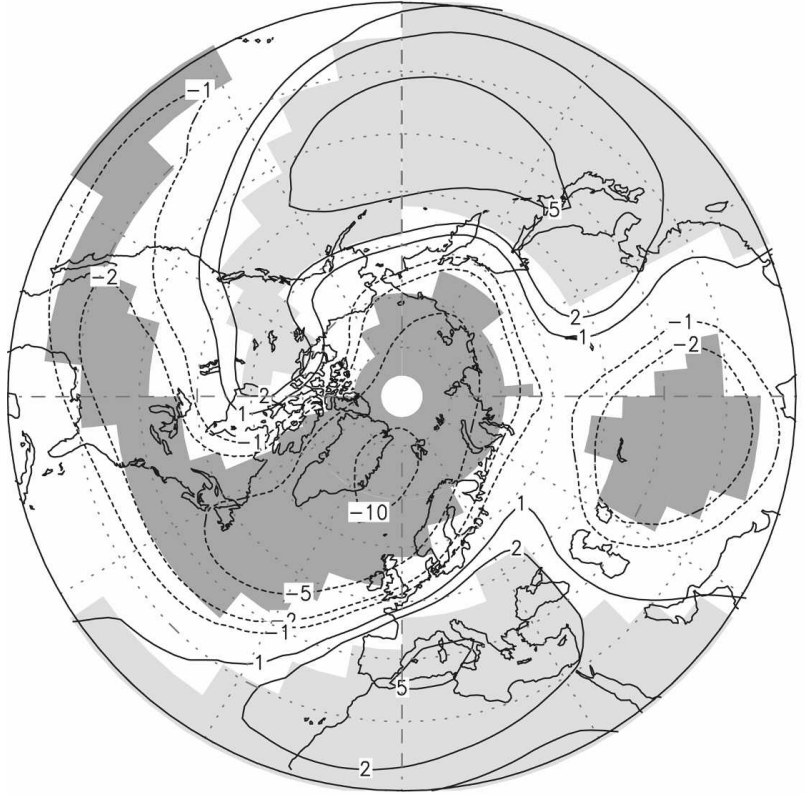

(c) ens. mean response to WTP forcing

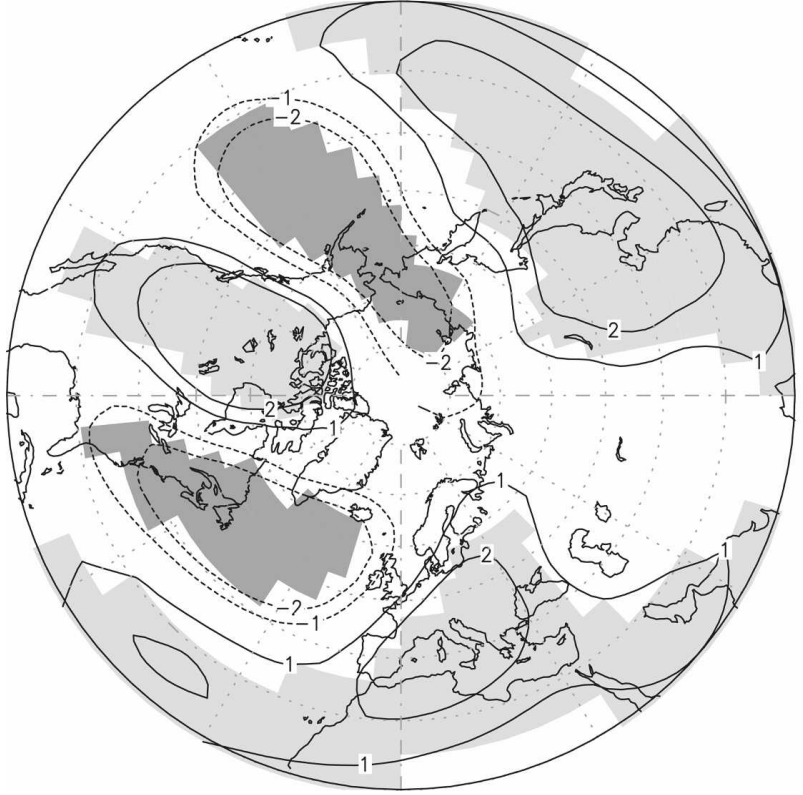

(b) to negative top level forcing

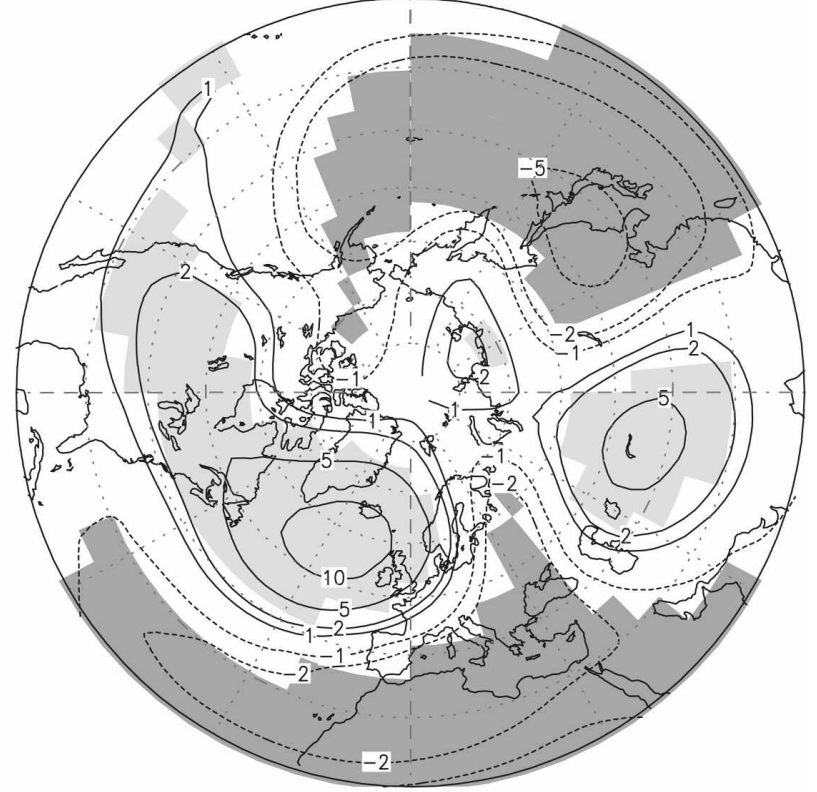

(d) to negative WTP forcing

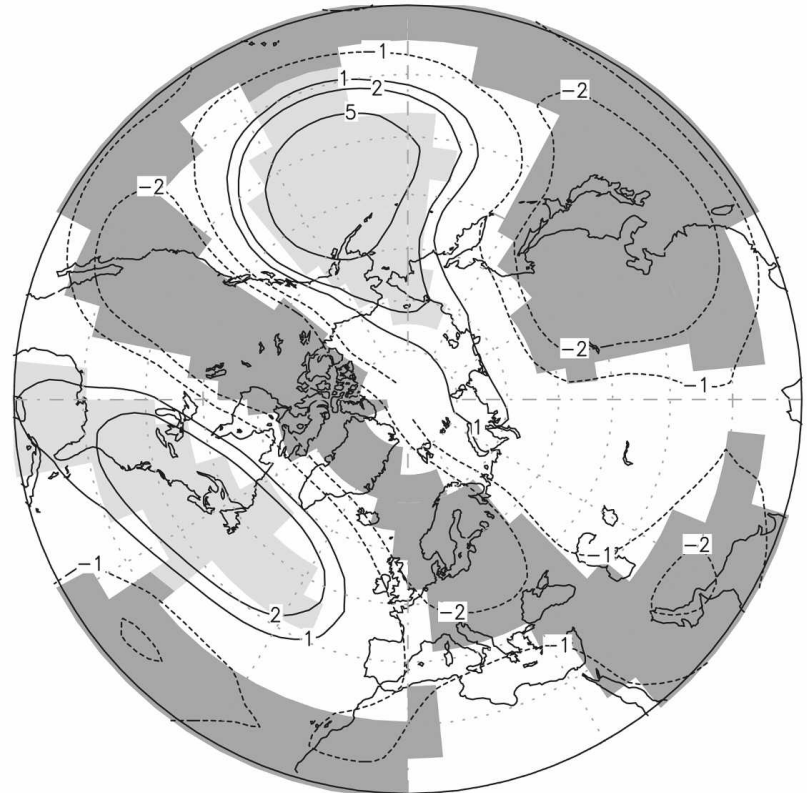

FIG. 11. As in Fig. 8 (nonlinear response to forcing derived from PUMA trajectories), but with the anomalous forcing restricted to (a) the top model level (about $100 \mathrm{hPa}$ ) and (c) the western tropical Pacific $\left(30^{\circ} \mathrm{S}-30^{\circ} \mathrm{N}\right.$ and $\left.120^{\circ}-210^{\circ} \mathrm{E}\right)$; (b) as in (a) but with reversed sign; and (d) as in (c) but with reversed sign.

linear model response, but with similar amplitude, and will not be discussed further here.

\section{Summary and discussion}

In this paper, we have applied a tangent linear adjoint model in an effort to clarify aspects of the forcing for the trend in the Northern Hemisphere winter tropospheric circulation, 1948-99. In particular, we have taken as a target pattern the 51-yr trend restricted to the region north of $20^{\circ} \mathrm{N}$, and asked what is the optimal forcing that excites this target with different lead times. The modeling system we use is PUMA, developed at the University of Hamburg (Fraedrich et al. 2005), and 
the setup and model parameters we use are the same as in Hall (2000) and Lu et al. (2004). In particular, we use T21 horizontal resolution with 5 levels in the vertical. In the fully nonlinear (forward) model runs, the forcing for the unperturbed control integration is the same as used in Hall (2000) and leads to a realistic Northern Hemisphere winter climate. In these runs, the optimally derived forcing is added as a perturbation to the climatological forcing using a range of amplitudes and lead times. For two different lead times ( 6 and 12 days), and for a range of both positive and negative amplitudes, we find that application of the derived forcing shows a systematic tendency (in an ensemble mean sense) to reproduce the target pattern (with negative amplitude if the forcing is negative). We also verified the derived forcing using forward runs with a model linearized about climatology, again showing the tendency of the model to produce the target pattern. This is true both for forcing derived from a model linearized about climatology, and for forcing that is the average of sensitivities derived from a tangent linear model in which the trajectories are taken either from observations (projected on the model grid) or from a nonlinear model run with climatological forcing. It is clear from the results that the essence of the forcing sensitivities is captured by the case linearized about climatology, although using averaged sensitivities from time-evolving trajectories of either observations or the nonlinear model generally simplifies the forcing sensitivities (e.g., by reducing the forcing sensitivity over the Asian continent and Africa, shown in Figs. 4a, 5a, and 6a).

The optimal forcing we have derived indicates the importance of forcing from the tropical Indo-Pacific region, as in Lu et al. (2004), especially for the deepening trend of the Aleutian low, consistent with Hurrell et al. (2004) and Hoerling et al. (2004). The optimally derived forcing for the top level is also suggestive of forcing for the northern annular mode (or Arctic Oscillation), related to influences from the stratosphere. By restricting the forcing to specific parts of the model domain, we are able to confirm the ability of forcing over the tropical Pacific region to force the North $\mathrm{Pa}$ cific part of the trend. We also find that the annular mode-type forcing is effective at exciting the trend pattern in the Euro-Atlantic/Asian sector. It is particularly interesting that a nonlinear model run, with the perturbed forcing applied only to the top model level, excites an annular mode-type response of similar amplitude and of the right sign as the observed trend in the Euro-Atlantic/Asian sector, but of opposite sign over the North Pacific sector. This result lends support to Scaife et al. (2005), who have argued an important role for the stratosphere for explaining the observed trend over the Euro-Atlantic sector. Furthermore, it is possible that the stratospheric trend itself could be a consequence of the upward trend in tropical SST during the last half of the twentieth century, rather than being of internal stratospheric origin, an issue for further study. If this is the case, then the stratosphere could act as a "bridge" linking forcing in the tropical Indo-Pacific region to the Euro-Atlantic/Asian sectors.

Finally we note that the adjoint approach outlined in this paper finds only the optimal forcing perturbation that gives a model response with a given projection on the target pattern. This means, for example, that forward model runs to which the derived forcing is applied are not guaranteed to reproduce the target pattern exactly, even when the forward model is linear (a good example can be seen by comparing Fig. $7 \mathrm{~b}$ with $7 \mathrm{a}$ ). More importantly, the derived forcing perturbation need not be representative of the forcing perturbation that actually generated the target pattern in nature. For example, Gritsun and Branstator (2007) find that a diabatic heating anomaly over the Indian Ocean is important for driving the positive phase of the northern annular mode in their model, a result that is consistent with the work of Hoerling et al. (2004). Yet such a diabatic heating anomaly is not extracted by our adjoint technique, suggesting that it is not "optimal." Nevertheless, we believe our work shows how a tangent linear adjoint can successfully be applied to the understanding of a climate response problem, even though the theoretical underpinning is linear with lead times of order 10 days.

Acknowledgments. S. Blessing has been supported from SFB 512, funded by the Deutsche Forschungsgemeinschaft. RJG received support from the Canadian CLIVAR Research Network funded by NSERC and CFCAS, and the NSERC/MARTEC/MSC Industrial Research Chair. The interpolated NCEP-NCAR reanalysis data and the Hall-model forcing were provided by Dr. Hai Lin at McGill University in Montreal, Canada. The authors are grateful to Dr. Jian Lu for his assistance with the handling of these data and the climatological forcing of the Hall model. We are also grateful to Dr. Torben Kunz, Dr. Jian Lu, and three anonymous reviewers for detailed and helpful comments on the manuscript.

\section{APPENDIX}

\section{Relationship between Sensitivities and Optimal Forcing}

The kinetic energy part of the energy matrix defined by Eq. (6) involves the transform of vorticity and di- 
vergence to the horizontal velocity components $u$ and $v$. Defining $\mathbf{U}$ as the operator $(\mathbf{k} \times \boldsymbol{\nabla}, \boldsymbol{\nabla})^{\mathrm{T}}$, which converts $(u, v)^{\mathrm{T}}$ to $(\zeta, D)^{\mathrm{T}}$, and $\mathbf{Z}=\mathbf{U}^{-1}=\mathbf{U} \Delta^{-1}$ as the inverse of $\mathbf{U}$ [as used for Eq. (6)], the following relationship holds for the optimal forcings in terms of velocities, $\mathbf{f}_{\text {opt }_{u, v}}$, and vorticity and divergence, $\mathbf{f}_{\text {opt }_{\zeta, \mathbf{D}}}$ :

$$
\mathbf{f}_{\text {opt }_{\zeta, D}}=\mathbf{U f}_{\text {opt }_{u, v}},
$$

and for the forcing sensitivities with respect to velocities $\mathbf{f}_{u, v}^{*}$, and vorticity and divergence $\mathbf{f}_{\zeta, D}^{*}$ (cf. appendix B of Kleist and Morgan 2005):

$$
\mathbf{f}_{u, v}^{*}=\mathbf{U} * \mathbf{f}_{\zeta, D}^{*} .
$$

Furthermore we can write the submatrix $\mathbf{E}_{\zeta, D}$ of $\mathbf{E}$, which operates on the components contributing to kinetic energy, as

$$
\mathbf{E}_{\zeta, D}=\mathbf{Z} * \mathbf{E}_{u, v} \mathbf{Z}
$$

since $\mathbf{W}$ is diagonal and $\left\langle\mathbf{Z} \cdot, \mathbf{W E}_{u, v} \mathbf{Z} \cdot\right\rangle=\left\langle\cdot, \mathbf{Z} * \mathbf{W} \mathbf{E}_{u, v}\right.$ $\mathbf{Z} \cdot\rangle$. Inserting Eq. (A3) into Eq. (9) yields

$$
\mathbf{f}_{\mathrm{opt}_{\zeta, D}}=\lambda \mathbf{U} \mathbf{E}_{u, v}^{-1} \mathbf{W}^{-1} \mathbf{U} * \mathbf{f}_{\zeta, D}^{*} .
$$

Since $\mathbf{E}_{u, v}$ and $\mathbf{W}$ are diagonal, we find the shape of $\mathbf{f}_{\zeta, D}^{*}$ and $\mathbf{f}_{\mathrm{opt}_{\zeta D}}$ to differ only from the combined operator UU*, which is equal to $-\mathbf{I} \Delta$ (I being the identity matrix). This reflects the difference between kinetic energy weights and enstrophy weights. The fact that the optimal forcing involves the application of a Laplacian operator to the forcing sensitivity explains why the former has much more small-scale structure than the latter.

\section{REFERENCES}

Bader, J., and M. Latif, 2003: The impact of decadal-scale Indian Ocean sea surface temperature anomalies on Sahelian rainfall and the North Atlantic Oscillation. Geophys. Res. Lett., 30, 2169, doi:10.1029/2003GL018426.

Baldwin, M. P., and T. J. Dunkerton, 1999: Propagation of the Arctic Oscillation from the stratosphere to the troposphere. J. Geophys. Res., 104, 30 937-30 946.

Barkmeijer, J., T. Iversen, and T. N. Palmer, 2003: Forcing singular vectors and other sensitive model structures. Quart. J. Roy. Meteor. Soc., 129, 2401-2423.

Branstator, G., 1985: Analysis of general circulation model seasurface temperature anomaly simulations using a linear model. Part I: Forced solutions. J. Atmos. Sci., 42, 2225-2241.

_ , and S. E. Haupt, 1998: An empirical model of barotropic atmosphere dynamics and its response to tropical forcing. $J$. Climate, 11, 2645-2667.

Charlton, A. J., A. O'Neill, W. A. Lahoz, and A. C. Massacand, 2004: Sensitivity of tropospheric forecasts to stratospheric initial conditions. Quart. J. Roy. Meteor. Soc., 130, 1771-1792.

Corti, S., and T. N. Palmer, 1997: Sensitivity analysis of atmospheric low-frequency variability. Quart. J. Roy. Meteor. Soc., 123, 2425-2447.

_, F. Molteni, and T. N. Palmer, 1999: Signature of recent cli- mate change in frequencies of natural atmospheric circulation regimes. Nature, 398, 799-802.

Deser, C., A. S. Phillips, and J. W. Hurrell, 2004: Pacific interdecadal climate variability: Linkages between the tropics and the North Pacific during boreal winter since 1900. J. Climate, 17, 3109-3124.

Errico, R. M., 2000: Interpretations of the total energy and rotational energy norms applied to determination of singular vectors. Quart. J. Roy. Meteor. Soc., 126, 1581-1599.

Eyink, G. L., T. W. N. Haine, and D. J. Lea, 2004: Ruelle's linear response formula, ensemble adjoint schemes and Lévy flights. Nonlinearity, 17, 1867-1889, doi:10.1088/0951-7715/17/ $5 / 016$.

Feldstein, S., 2002: The recent trend and variance increase of the annular mode. J. Climate, 15, 88-94.

Fraedrich, K., E. Kirk, U. Luksch, and F. Lunkeit, 2005: The Portable University Model of the Atmosphere (PUMA): Storm track dynamics and low-frequency variability. Meteor. Z., 14, 735-745.

Giering, R., and T. Kaminski, 1998: Recipes for adjoint code construction. ACM Trans. Math. Software, 24, 437-474, doi:10.1145/293686.293695.

Gillett, N. P., and D. W. J. Thompson, 2003: Simulation of recent Southern Hemisphere climate change. Science, 302, 273-275.

- H. F. Graf, and T. J. Osborn, 2003: Climate change and the North Atlantic Oscillation. The North Atlantic Oscillation: Climatic Significance and Environmental Impact, Geophys. Monogr., Vol. 134, Amer. Geophys. Union, 193-209.

Greatbatch, R. J., 2000: The North Atlantic Oscillation. Stochastic Environ. Res. Risk Assess., 14, 213-242.

, and T. Jung, 2007: Local versus tropical diabatic heating and the winter North Atlantic Oscillation. J. Climate, 20, 20582075.

Gritsun, A., and G. Branstator, 2007: Climate response using a three-dimensional operator based on the fluctuation dissipation theorem. J. Atmos. Sci., 64, 2558-2575.

Hall, N. M. J., 2000: A simple GCM based on dry dynamics and constant forcing. J. Atmos. Sci., 57, 1557-1572.

Hoerling, M. P., J. W. Hurrell, and T. Xu, 2001: Tropical origins for recent North Atlantic climate change. Science, 292, 90-92.

,,-- T. Xu, G. T. Bates, and A. S. Phillips, 2004: Twentieth century North Atlantic climate change. Part II: Understanding the effect of Indian Ocean warming. Climate Dyn., 23, 391-405.

Hoskins, B. J., and A. J. Simmons, 1975: A multi-layer spectral model and the semi-implicit method. Quart. J. Roy. Meteor. Soc., 101, 637-655.

Hurrell, J. W., 1996: Influence of variations in extratropical wintertime teleconnections on Northern Hemisphere temperature. Geophys. Res. Lett., 23, 665-668.

— Y. Yushnir, M. Visbeck, and G. Ottersen, 2003: An overview of the North Atlantic Oscillation. The North Atlantic Oscillation: Climatic Significance and Environmental Impact, Geophys. Monogr., Vol. 134, Amer. Geophys. Union, 1-35.

—, M. P. Hoerling, A. S. Phillips, and T. Xu, 2004: Twentieth century North Atlantic climate change. Part I: Assessing determinism. Climate Dyn., 23, 371-389.

Iversen, T., J. Kristiansen, T. Jung, and J. Barkmeijer, 2008: Optimal atmospheric forcing perturbations for the Cold Ocean Warm Land pattern. Tellus, 60A, 528-546.

Jung, T., and J. Barkmeijer, 2006: Sensitivity of the tropospheric 
circulation to changes in the strength of the stratospheric polar vortex. Mon. Wea. Rev., 134, 2191-2207.

Kleist, D. T., and M. Morgan, 2005: Interpretation of the structure and evolution of adjoint-derived forecast sensitivity gradients. Mon. Wea. Rev., 133, 466-484.

Kucharski, F., F. Molteni, and A. Bracco, 2006: Decadal interactions between the western tropical Pacific and the North Atlantic Oscillation. Climate Dyn., 26, 79-91, doi:10.1007/ s00382-005-0085-5.

Langland, R., M. A. Shapiro, and R. Gelaro, 2002: Initial condition sensitivity and error growth in forecasts of the 25 January 2000 east coast snowstorm. Mon. Wea. Rev., 130, 957-974.

Lu, J., R. J. Greatbatch, and K. A. Peterson, 2004: Trend in Northern Hemisphere winter atmospheric circulation during the last half of the twentieth century. J. Climate, 17, 37453760 .

Marshall, J., and F. Molteni, 1993: Toward a dynamical understanding of planetary-scale flow regimes. J. Atmos. Sci., 50, 1792-1818.

Penland, C., 1989: Random forcing and forecasting using principal oscillation pattern analysis. Mon. Wea. Rev., 117, 2165-2185.

Polvani, L. M., and P. J. Kushner, 2002: Tropospheric response to stratospheric perturbations in a relatively simple general circulation model. Geophys. Res. Lett., 29, 1114, doi:10.1029/ 2001GL014284.

Sardeshmukh, P. D., and B. J. Hoskins, 1988: The generation of global rotational flow by steady idealized tropical divergence. J. Atmos. Sci., 45, 1228-1251.

Scaife, A. A., J. R. Knight, G. K. Vallis, and C. K. Folland, 2005: A stratospheric influence on the winter NAO and North At- lantic surface climate. Geophys. Res. Lett., 32, L18715, doi:10.1029/2005GL023226.

Taguchi, M., and D. L. Hartmann, 2006: Increased occurrence of stratospheric sudden warmings during El Niño as simulated by WACCM. J. Climate, 19, 324-332.

Talagrand, O., 1991: The use of adjoint equations in numerical modeling of the atmospheric circulation. Automatic Differentiation of Algorithms: Theory, Implementation, and Application, A. Griewank and G. Corlies, Eds., Society for Industrial and Applied Mathematics, 169-180.

Thompson, D. W. J., and J. M. Wallace, 2000: Annular modes in the extratropical circulation. Part I: Month-to-month variability. J. Climate, 13, 1000-1016.

,-- , and G. C. Hegerl, 2000: Annular modes in the extratropical circulation. Part II: Trends. J. Climate, 13, 1018-1036.

Trenberth, K. E., J. M. Caron, D. P. Stepaniak, and S. Worley, 2002: Evolution of El Niño-Southern Oscillation and global atmospheric surface temperatures. J. Geophys. Res., 107, 4065, doi:10.1029/2000JD000298.

Wallace, J. M., and D. S. Gutzler, 1981: Teleconnections in the geopotential height field during the northern hemisphere winter. Mon. Wea. Rev., 109, 784-812.

—, Y. Zhang, and L. Bajuk, 1996: Interpretation of decadal trends in Northern Hemisphere surface air temperature. $J$. Climate, 9, 249-259.

Wu, Q., and D. M. Straus, 2004: AO, COWL, and observed climate trends. J. Climate, 17, 2139-2156.

Wunsch, C., 1999: The interpretation of short climate records, with comments on the North Atlantic and Southern Oscillations. Bull. Amer. Meteor. Soc., 80, 245-255. 